\title{
Shape dynamics, lipid hydrodynamics, and the complex viscoelasticity of bilayer membranes
}

\author{
Mohammad Rahimi and Marino Arroyo* \\ Departament de Matemàtica Aplicada III, LaCàN, Universitat Politècnica de Catalunya (UPC), Barcelona 08034, Spain
}

(Received 25 April 2012; published 31 July 2012; corrected 8 August 2012)

\begin{abstract}
Biological membranes are continuously brought out of equilibrium, as they shape organelles, package and transport cargo, or respond to external actions. Even the dynamics of plain lipid membranes in experimental model systems are very complex due to the tight interplay between the bilayer architecture, the shape dynamics, and the rearrangement of the lipid molecules. We formulate and numerically implement a continuum model of the shape dynamics and lipid hydrodynamics, which describes the bilayer by its midsurface and by a lipid density field for each monolayer. The viscoelastic response of bilayers is determined by the stretching and curvature elasticity, and by the inter-monolayer friction and the membrane interfacial shear viscosity. While the bilayer equilibria are well understood theoretically, dynamical calculations have relied on simplified continuum approaches of uncertain transferability, or on molecular simulations reaching very limited length and time scales. Our approach incorporates the main physics, is fully nonlinear, does not assume predefined shapes, and can access a wide range of time and length scales. We validate it with the well understood tether extension. We investigate the tubular lipid transport between cells, the dynamics of bud absorption by a planar membrane, and the fate of a localized lipid density asymmetry in vesicles. These axisymmetric examples bear biological relevance and highlight the diversity of dynamical regimes that bilayers can experience.
\end{abstract}

DOI: 10.1103/PhysRevE.86.011932

PACS number(s): 87.16.dj, 87.16.A-, 87.16.dm

\section{INTRODUCTION}

Lipid bilayers are quasi-two-dimensional systems made out of two monolayers of lipid molecules held together by the hydrophobic effect. They form most biological containers in the cell, and exhibit a wide range of morphologies, from vesicles to tubes, including sheets or complex networks in the endoplasmic reticulum [1]. Bilayers behave as elastic membranes forming relatively stable structures. At the same time, they exhibit in-plane fluidity, essential to the motion of membrane proteins [2], or to the transport of lipids between cells through membrane tubes [3]. Besides lipid flows at fixed membrane shape, this malleability also allows bilayers to rapidly adapt their shape to accomplish important biological functions, such as vesicular transport in secretion [4], or area regulation in confined cells [5]. The goal of the present paper is to formulate and numerically implement a dynamical quantitative model for bilayers, observing the intimately coupled lipid hydrodynamics and shape dynamics.

Although a simple elastic surface model such as that described by the Helfrich curvature energy has been able to explain a number of observations of bilayer vesicles [6], it has long been recognized that in many situations the mechanical behavior of lipid membranes can only be explained by acknowledging its bilayer architecture [7]. Besides curvature elasticity, a general model for the energetics of bilayers [8] includes the stretching elasticity of each monolayer, which despite the generally small density deviations from equilibrium has a non-negligible effect. Physically, increasing the lipid density in one leaflet leads to a free energy penalty due to the steric interaction between the lipids, while decreasing it exposes the hydrophobic core, resulting again in a free energy cost. What makes bilayers very special mechanical systems is the in-plane fluidity of the monolayers, and their ability

*marino.arroyo@upc.edu to slip over each other. In physiological conditions, in-plane and inter-monolayer shear stresses are purely viscous, with experimentally characterized membrane shear viscosity and inter-monolayer friction coefficients [9].

In equilibrium, the bilayer continuum model gives rise a hierarchy of area difference elasticity models [8]. Yet, the membrane biophysics is fundamentally out of equilibrium. Biophysical experiments vividly exemplify nonequilibrium dynamics involving lipid density changes, such as the shape changes due to lipid translocation under the action of flippases [10], or a local $\mathrm{pH}$ disturbance, changing the preferred area per lipid [11,12]. Putting together the curvature and density elastic forces and the dissipative forces due to inter-monolayer friction, the membrane, and the surrounding bulk fluid shear viscosity, it is possible to derive a dynamical model for the shape and the monolayer density. Yet, such model is mathematically complex, and has only been formulated and exercised in simplified settings. The thermal undulations of bilayers are generally understood in terms of a linearized version of this model, considering infinitesimal shape and density perturbations around a planar bilayer at equilibrium [13]. Another important application of this theory, under the strong assumption of fixed shape, is in the hydrodynamics of tether extension [14]. This reference highlights the role of inter-monolayer drag for membrane flows around sharp geometries. The coupled lipid density and shape dynamics under finite perturbations have been analyzed in Ref. [15] for a predefined family of shapes and density distributions.

The complex equations for the dynamics of bilayers can be further simplified under specific conditions. In the simplest situation, the bilayer architecture and the internal dissipative mechanisms can be neglected for giant unilamellar vesicles, governed by capillarity, curvature elasticity, and bulk dissipation. Around sharp geometries, or for short wavelength thermal undulations, the inter-monolayer friction dominates the dissipation, and membrane and bulk shear viscosities may be neglected. There is a general tendency in the literature to 
neglect the membrane shear dissipation, notoriously difficult to treat mathematically [16], e.g., on the basis of scaling arguments involving the Saffman-Delbrück length scale. We show here and elsewhere [17] that such arguments, as well as the linearization of the equations around a planar configuration [13], can severely underestimate the role of membrane shear viscosity for significantly curved bilayers. Bilayers can also be studied theoretically with molecular dynamics. However, due to the slow relaxation times of bending modes [18], these methods are severely limited to small time and length scales [19,20].

In summary, lipid bilayers exhibit subtle mechanics, described by complex continuum equations, which have been judiciously simplified in some situations. Our goal here is to introduce a general mathematical model and its numerical implementation to deal with finite shape and density deviations, accounting for the bilayer nature of the system, its elasticity, and its internal dissipative mechanisms. We leave aside the extensively studied effect of the surrounding bulk fluid, which can be safely ignored for lipid bilayer systems smaller than a few microns, and specialize in the simulations to axisymmetric systems, allowing us to describe many of the important shapes bilayers adopt. Our model can describe complex lipid hydrodynamics mobilizing the inter-monolayer friction, such as flows around sharp geometries or as a result of localized density

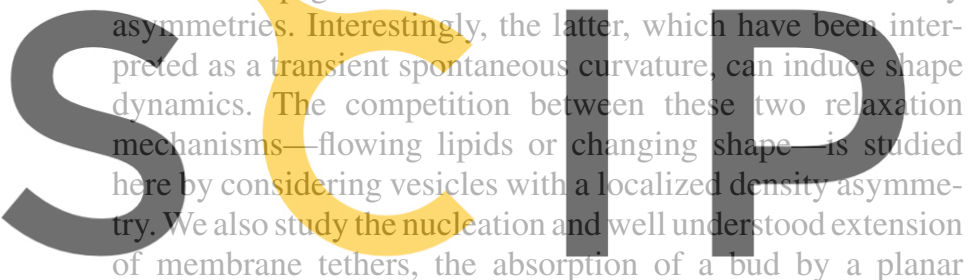
bilayer, and the recently observed tension-driven lipid flows

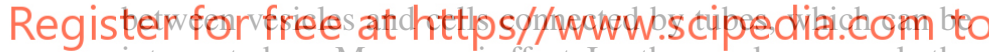
interpreted as a Marangoni effect. In other works, we apply the proposed model to confined bilayers [21], and to study proteinmembrane interactions. These examples illustrate the diversity and complexity of the bilayer dynamics, which make it difficult to provide accurate estimations based on simple arguments.

Section II describes the kinematics of our continuum model, which challenge traditional Lagrangian or Eulerian descriptions [22], the continuity equation, and the balance of linear momentum. The numerical implementation for axisymmetric bilayers, relying on a $B$-spline Galerkin method, is given in Sec. III. Section IV describes numerical calculations based on the proposed model, and Sec. V collects some concluding remarks.

\section{CONTINUUM MODEL}

We describe the configuration of the bilayer in terms of its midsurface and two lipid density fields, one for each monolayer, thus forcing at the outset the shape coupling of the two monolayers. The rate of change of the system is given by the rate of change of the midsurface, i.e., its normal velocity, and by the tangential velocity of the lipids of each monolayer, which are in general different, producing inter-monolayer slippage and friction. The lipid densities, and the tangential and normal velocities, are coupled through the continuity equation, expressing locally balance of mass. The governing equations are completed with balance of linear momentum, obtained with variational methods, where we ignore inertial forces given the low Reynolds numbers typical in bilayer mechanics. We pay particular attention to the kinematics, which are necessarily Lagrangian (tracking material particles) in the normal direction to the bilayer, but which cannot be Lagrangian tangentially due to inter-monolayer slippage. We propose an arbitrarily Lagrangian-Eulerian formulation, very useful in the numerical implementation, and provide detailed and accessible expressions for axisymmetric bilayers.

\section{A. Kinematics}

We consider the parametric description $x\left(\xi^{1}, \xi^{2}, t\right)$ of a bilayer midsurface $\Gamma_{t}$. As argued above, a point $\left(\xi^{1}, \xi^{2}\right)$ in parameter space cannot label a material particle, and the time derivative of this parametrization is not in general the velocity of material particles. We express it as

$$
x_{, t}=W=w+v_{n} n,
$$

where the normal velocity $v_{n}$ has mechanical relevance, since it characterizes the rate of shape changes, whereas the tangential field $w$ is the velocity of points with fixed parametric coordinates. We denote by $g_{a b}=x_{, a} \cdot x_{, b}$ the metric tensor, by $n$ the unit normal to the surface, and by $k=-\nabla n$ the

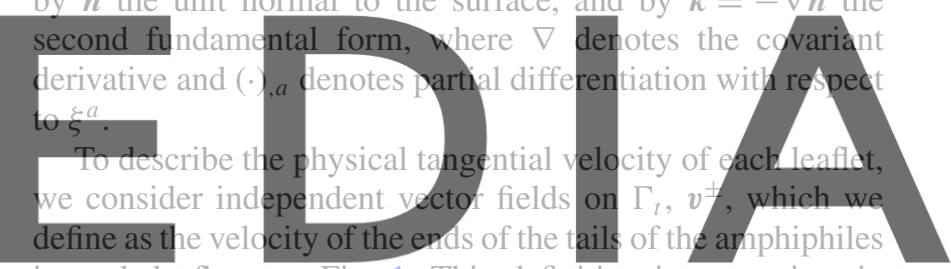
in each leaflet; see Fig. 1. This definition is convenient in the formulation of the inter-monolayer friction. For numerical douvenliard thellwe rsigendaithgentiatherwaterthark parametrization, and formulate the mechanics covariantly in this respect. Such a description of motion is often referred to as arbitrarily Lagrangian-Eulerian (ALE) kinematics; see Refs. [22,23] for details. The tangential convective velocity, i.e., the tangential physical velocity relative to points of fixed parameter value $\left(\xi^{1}, \xi^{2}\right)$, is $\boldsymbol{c}^{ \pm}=\boldsymbol{v}^{ \pm}-\boldsymbol{w}$. If $\boldsymbol{w}=0$, we recover an Eulerian description in the tangential direction. Lagrangian descriptions are characterized by zero convective velocity. It is clear that we cannot recover a purely Lagrangian formulation since in general the leaflets slip relative to each other, and therefore $\boldsymbol{w}$ cannot equal $\boldsymbol{v}^{+}$and $\boldsymbol{v}^{-}$at the same time.

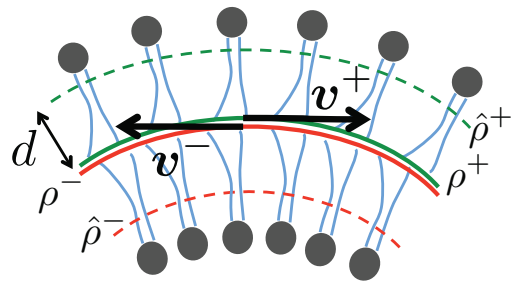

FIG. 1. (Color online) Description of a bilayer membrane. $\boldsymbol{v}^{ \pm}$ denotes the tangential velocity field of each monolayer on the midsurface. The density field on the neutral surface of each monolayer $\hat{\rho}^{ \pm}$, and the projected density field on the midsurface $\rho^{ \pm}$are also shown. 


\section{B. Continuity equation}

Following Ref. [8], we describe each leaflet by a neutral surface representative of its mechanics, i.e., the density of the lipids in this surface $\hat{\rho}^{ \pm}$is the order parameter that allows us to define the areal elastic energy. The projected densities onto the midsurface of the bilayer $\rho^{ \pm}$, see Fig. 1, deviate from $\hat{\rho}^{ \pm}$ according to the following relation:

$$
\hat{\rho}^{ \pm}=\rho^{ \pm}\left[1 \mp 2 d H+O\left(d^{2} K\right)\right] .
$$

where $d$ is the distance between the bilayer midsurface and the neutral surface of the monolayers, usually located close to the polar-apolar interface [24], $H$ denotes the mean curvature (half the trace of the second fundamental form), and $K$ denotes the Gaussian curvature (the determinant of the second fundamental form). A convenient approximation, for small deviations from a reference density of linids $\rho_{0}$, is

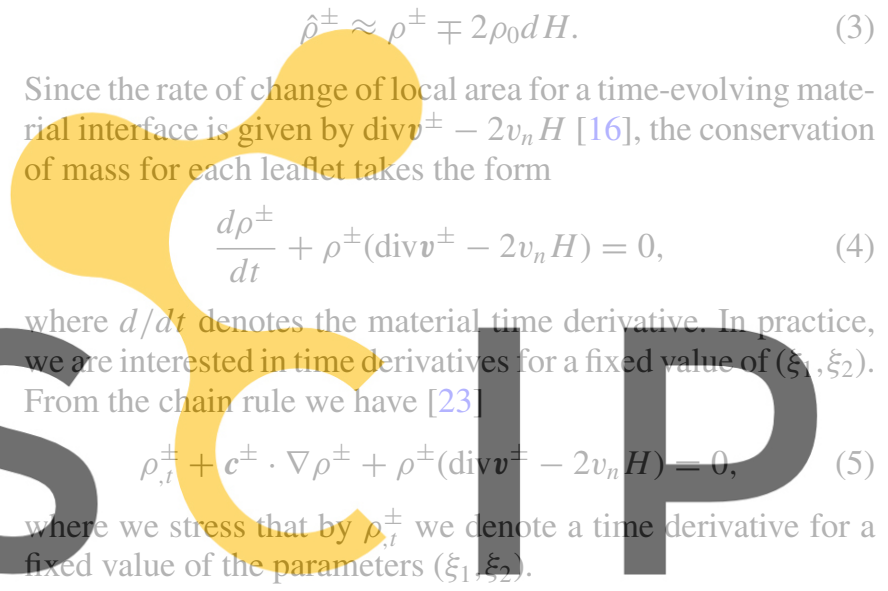

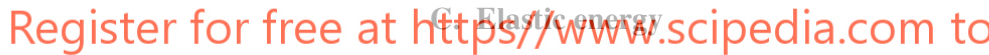

Deviations from the equilibrium density $\rho_{0}$ result in excess elastic energy. Additionally, each leaflet stores elastic energy when curved, for which we follow the classic Helfrich functional ignoring the Gaussian curvature term for simplicity. Consequently the total elastic energy can be written as [8]

$$
\begin{aligned}
\Pi\left[\boldsymbol{x}, \rho^{ \pm}\right]= & \int_{\Gamma} \frac{K_{s}}{2}\left[\left(\frac{\hat{\rho}^{+}}{\rho_{0}}-1\right)^{2}+\left(\frac{\hat{\rho}^{-}}{\rho_{0}}-1\right)^{2}\right] d S \\
& +\int_{\Gamma} \frac{\kappa}{2}\left(2 H-C_{0}\right)^{2} d S,
\end{aligned}
$$

where $K_{s}$ is the elastic stretching modulus of each monolayer, $\kappa$ is the bending modulus, and $C_{0}$ is the spontaneous curvature. We rewrite the total elastic energy by substituting Eq. (3),

$$
\begin{aligned}
\Pi\left[\boldsymbol{x}, \rho^{ \pm}\right]= & \int_{\Gamma} \frac{K_{s}}{2}\left(\frac{\rho^{ \pm}}{\rho_{0}}-1 \mp 2 d H\right)^{2} d S \\
& +\int_{\Gamma} \frac{\kappa}{2}\left(2 H-C_{0}\right)^{2} d S .
\end{aligned}
$$

Here and in subsequent expressions we imply a summation of the "+" and "-" contributions. The interested reader can find in Appendix A the expression of the elastic surface stress tensor derived from this Hamiltonian.

We compute now the rate of change of the elastic energy functional, required to derive the governing equations. The resulting expression should be independent of the parametrization of the midsurface, and hence should depend on $\boldsymbol{x}_{, t}$ through $v_{n}$ alone. By using the continuity equation, we express this functional in terms of the velocities $v_{n}$ and $\boldsymbol{v}^{ \pm}$, instead of $v_{n}$ and $\rho_{t}^{ \pm}$. For convenience, we consider in this derivation an Eulerian gauge, for which the rate of change of the area element is $-2 v_{n} H d S$, and the rate of change of the mean curvature follows the classical expression $2 H_{, t}=$ $\Delta v_{n}+v_{n}\left(4 H^{2}-2 K\right)$. Using these two expressions, Eq. (5) with $\boldsymbol{c}^{ \pm}=\boldsymbol{v}^{ \pm}$, and rearranging terms, we find $\dot{\Pi}=\dot{\Pi}_{\kappa}+\dot{\Pi}_{K_{s}}$ with

$$
\dot{\Pi}_{\kappa}\left[v_{n}\right]=\kappa \int_{\Gamma}\left(2 H-C_{0}\right)\left\{\Delta v_{n}+\left(2 H^{2}-2 K+H C_{0}\right) v_{n}\right\} d S,
$$

and

$$
\begin{aligned}
\dot{\Pi}_{K_{s}}\left[v_{n}, v^{ \pm}\right] & \\
= & K_{s} \int_{\Gamma}\left(\frac{\rho^{ \pm}}{\rho_{0}}-1 \mp 2 d H\right)\left\{-\operatorname{div}\left(\frac{\rho^{ \pm}}{\rho_{0}} v^{ \pm}\right)\right. \\
& \left.+v_{n} H\left(\frac{\rho^{ \pm}}{\rho_{0}}+1\right) \mp d\left[\Delta v_{n}+2 v_{n}\left(H^{2}-K\right)\right]\right\} d S,
\end{aligned}
$$

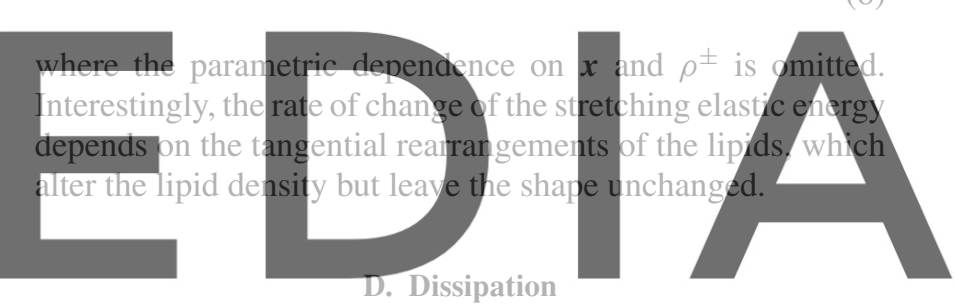

The internal dissipative mechanisms of the bilayer include

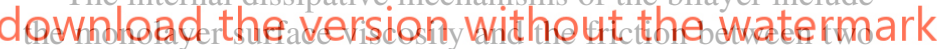
monolayers. We consider each monolayer as a Newtonian interfacial fluid [25], which can only support a tangential viscous stress

$$
\sigma_{\|}^{\mathrm{visc}, \pm}=2 \mu \boldsymbol{d}^{ \pm}+\lambda\left(\operatorname{tr} \boldsymbol{d}^{ \pm}\right) \boldsymbol{g},
$$

where $\mu$ and $\lambda$ are the monolayer shear and dilatational viscosities (see Ref. [9] for measured coefficients), and the rate-of-deformation tensor $\boldsymbol{d}$ includes a contribution from the normal velocity of the interface in the presence of curvature,

$$
\boldsymbol{d}^{ \pm}=\frac{1}{2}\left[\boldsymbol{\nabla} \boldsymbol{v}^{ \pm}+\left(\boldsymbol{\nabla} \boldsymbol{v}^{ \pm}\right)^{T}\right]-v_{n} \boldsymbol{k}
$$

Assuming as usual a linear relation between the intermonolayer shear traction and the slippage velocity $\boldsymbol{\tau}=$ $b_{m}\left(\boldsymbol{v}^{+}-\boldsymbol{v}^{-}\right)$, we can write the Rayleigh dissipation potential from which all these viscous stresses derive:

$$
\begin{aligned}
W\left[v_{n}, \boldsymbol{v}^{ \pm}\right]= & W_{\mu}\left[v_{n}, \boldsymbol{v}^{ \pm}\right]+W_{b_{m}}\left[\boldsymbol{v}^{ \pm}\right] \\
= & \frac{1}{2} \int_{\Gamma}\left(2 \mu \boldsymbol{d}: \boldsymbol{d}+\lambda(\operatorname{tr} \boldsymbol{d})^{2}\right)^{ \pm} d S \\
& +\frac{b_{m}}{2} \int_{\Gamma}\left\|\boldsymbol{v}^{+}-\boldsymbol{v}^{-}\right\|^{2} d S .
\end{aligned}
$$

See Ref. [16] for a detailed account of the membrane dissipation. 


\section{E. Governing equations}

The dynamics of the system can be obtained by minimizing the Rayleigh dissipation potential plus the rate of change of the elastic energy with respect to the variables expressing the rate of change of the system [26]. This minimization is often constrained, for instance by boundary conditions or a fixed enclosed volume. For this purpose, we form the Lagrangian

$\mathcal{L}\left[v_{n}, \boldsymbol{v}^{ \pm}, \boldsymbol{\Lambda}\right]=W\left[v_{n}, \boldsymbol{v}^{ \pm}\right]+\dot{\Pi}\left[v_{n}, \boldsymbol{v}^{ \pm}\right]-\boldsymbol{\Lambda} \cdot \boldsymbol{C}\left[v_{n}, \boldsymbol{v}^{ \pm}\right]$,

where $\boldsymbol{C}\left[v_{n}, \boldsymbol{v}^{ \pm}\right]$collects all the constraints and $\boldsymbol{\Lambda}$ the corresponding Lagrange multipliers. For instance, a fixed enclosed volume constraint for a closed bilayer is expressed as $\int_{\Gamma} v_{n} d S=0$, and the associate Lagrange multiplier is the hydrostatic pressure jump across the bilayer. The governing equations are then obtained by making the Lagrangian stationary for all admissible variations

$$
\delta_{v_{n}} \mathcal{L}=\delta_{v^{+}} \mathcal{L}=\delta_{v^{-}} \mathcal{L}=\delta_{\Lambda} \mathcal{L}=0,
$$

which is a form of the principle of virtual power. It is straightforward to include external forces such as a prescribed membrane tension at the boundary of a domain. From these equations, by integration by parts, the Euler-Lagrange equations can be derived by a direct calculation. The only term involving complex calculations is the membrane dissipation; see Ref. [16].
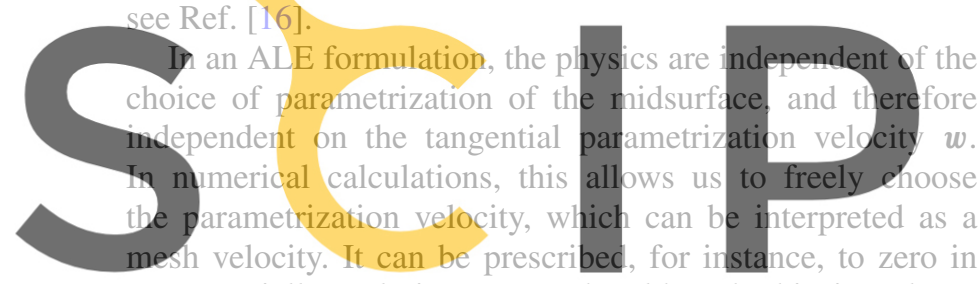

a tangentially Eulerian approach, although this introduces numerical stiffness in the equations. It is alsq.possible to

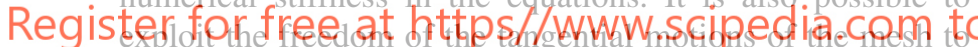

adapt to discretization density to the features of the solution, although we do not attempt this here. Instead, we view the mesh velocity as an additional unknown. To break the invariance of the physical equations with respect to $w$, we include a functional to damp the mesh velocity,

$$
\widehat{W}[\boldsymbol{w}]=\frac{\hat{\mu}}{2 S} \int_{\Gamma}|\boldsymbol{w}|^{2} d S,
$$

where $\hat{\mu}$ is a numerical parameter with units of physical viscosity and $S$ is the bilayer surface area. This functional can be understood as a penalty approach to enforce approximately the Eulerian gauge.

Once the lipid, shape, and parametrization velocities have been obtained from these equations, one can integrate in time the parametrization from Eq. (1), and monolayer densities from the local statement of balance of mass in Eq. (5).

\section{F. Particularization to axisymmetric surfaces}

We consider the parametric description of an axisymmetric surface in terms of the generating curve, i.e., the surface $\Gamma_{t}$ at a given instant $t$ by

$$
\boldsymbol{x}(u, \theta, t)=\{r(u, t) \cos \theta, r(u, t) \sin \theta, z(u, t)\},
$$

where $u \in[0,1], \theta \in[0,2 \pi]$, and $\{r(u, t), z(u, t)\}$ is the parametric description of the generating curve. The velocity of the parametrization is

$$
\left\{r_{, t}, z_{, t}\right\}=w \boldsymbol{t}+v_{n} \boldsymbol{n},
$$

where $\boldsymbol{t}=1 / a\left\{r^{\prime}, z^{\prime}\right\}$ is a tangent unit vector to the generating curve, $\boldsymbol{n}=1 / a\left\{-z^{\prime}, r^{\prime}\right\}$ is a unit normal, and $a(u)=$ $\sqrt{\left[r^{\prime}(u)\right]^{2}+\left[z^{\prime}(u)\right]^{2}}$ is the norm of the curve speed. Throughout the paper, $(\cdot)^{\prime}$ denotes partial differentiation with respect to the parameter $u$. From the above relations, we have

$$
w=\frac{1}{a}\left(r^{\prime} r_{, t}+z^{\prime} z_{, t}\right), \quad v_{n}=\frac{1}{a}\left(-z^{\prime} r_{, t}+r^{\prime} z_{, t}\right) .
$$

We assume that the tangential velocity of the lipids in each monolayer does not have azimuthal components, i.e., $\boldsymbol{v}^{ \pm}=$ $v^{ \pm} \boldsymbol{t}$.

For axisymmetric surfaces, integrals on the surface can be brought to the interval $[0,1]$ with the relation $d S=(2 \pi$ ar $) d u$. Following Refs. [16,27], the rate-of-deformation tensor can be written in an orthonormal coordinate system with basis vectors along the generating curves and the parallels of the surface as

$$
d^{ \pm}=\frac{1}{a}\left[\begin{array}{cc}
v^{ \pm \prime} & 0 \\
0 & v^{ \pm} r^{\prime} / r
\end{array}\right]-\frac{v_{n}}{a}\left[\begin{array}{cc}
b / a^{2} & 0 \\
0 & z^{\prime} / r
\end{array}\right],
$$

where $b(u)=-r^{\prime \prime}(u) z^{\prime}(u)+r^{\prime}(u) z^{\prime \prime}(u)$. The mean and Gaus-

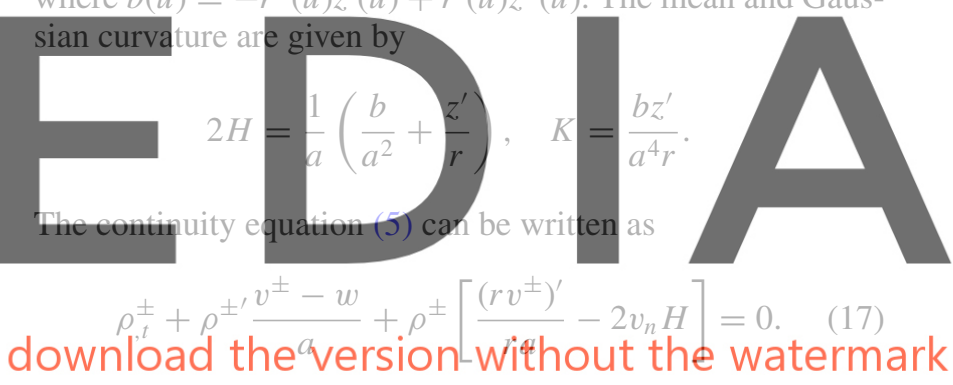

Specializing Eq. (11) in the present setting, the dissipation potentiais can be written concisely as

$$
W_{b_{m}}\left[v^{ \pm}\right]=\frac{b_{m}}{2} \int_{0}^{1}\left[v^{+}, v^{-}\right]\left[\begin{array}{cc}
1 & -1 \\
-1 & 1
\end{array}\right]\left[\begin{array}{l}
v^{+} \\
v^{-}
\end{array}\right](2 \pi a r) d u
$$

and

$$
W_{\mu}\left[r_{, t}, z_{, t}, v^{ \pm}\right]=\frac{1}{2} \int_{0}^{1} \mathbf{U}^{T} \mathbf{A} \mathbf{U}(2 \pi a r) d u,
$$

where $\mathbf{U}^{T}=\left[r_{, t}, z_{, t}, v^{+}, v^{-}, v^{+^{\prime}}, v^{-\prime}\right]$ and $\mathbf{A}$ is a symmetric matrix given in Appendix B.

The parametrization dissipation potential takes the simple form

$$
\widehat{W}\left[r_{, t}, z_{, t}\right]=\frac{\hat{\mu}}{S} \int_{0}^{1} \frac{\pi r}{a}\left[r_{, t}, z_{, t}\right]\left[\begin{array}{cc}
r^{\prime 2} & r^{\prime} z^{\prime} \\
r^{\prime} z^{\prime} & z^{\prime 2}
\end{array}\right]\left[\begin{array}{l}
r_{, t} \\
z_{, t}
\end{array}\right] d u .
$$

Similarly we implement the axisymmetric version of the elastic energy. Rather than using the general expression in the previous section for the rate of change of the elastic energy, we find a numerically more convenient expression avoiding third-order derivatives next. By taking variations directly of 
the axisymmetric functional

$$
\begin{aligned}
\Pi\left[r, z, \rho^{ \pm}\right]= & \int_{0}^{1} \frac{K_{s}}{2}\left(\frac{\rho^{ \pm}}{\rho_{0}} \mp 2 d H-1\right)^{2}(2 \pi a r) d u \\
& +\int_{0}^{1} \frac{\kappa}{2}\left(2 H-C_{0}\right)^{2}(2 \pi a r) d u,
\end{aligned}
$$

we obtain

$$
\begin{aligned}
\dot{\Pi}\left[r_{, t}, z_{, t}, \dot{\rho}^{ \pm}\right]= & \int_{0}^{1} K_{s}\left[\left(\mp 2 d H_{, t}+\frac{\rho_{, t}^{ \pm}}{\rho_{0}}\right)\left(\frac{\rho^{ \pm}}{\rho_{0}} \mp 2 d H-1\right)\right. \\
& \left.+2 \kappa\left(2 H-C_{0}\right) H_{, t}\right](2 \pi a r) d u \\
& +\int_{0}^{1}\left[\frac{K_{s}}{2}\left(\frac{\rho^{ \pm}}{\rho_{0}} \mp 2 d H-1\right)^{2}\right. \\
& \left.+\frac{\kappa}{2}\left(2 H-C_{0}\right)^{2}\right]\left(a_{, t} r+a r_{, t}\right) 2 \pi d u,
\end{aligned}
$$

where $H_{, t}, a_{, t}$, and $\rho_{. t}^{ \pm}$are given in Appendix C. Using the continuity equation, $\dot{\Pi}$ becomes a functional of velocities only. Despite the long expressions, this functional has a simple structure of the form

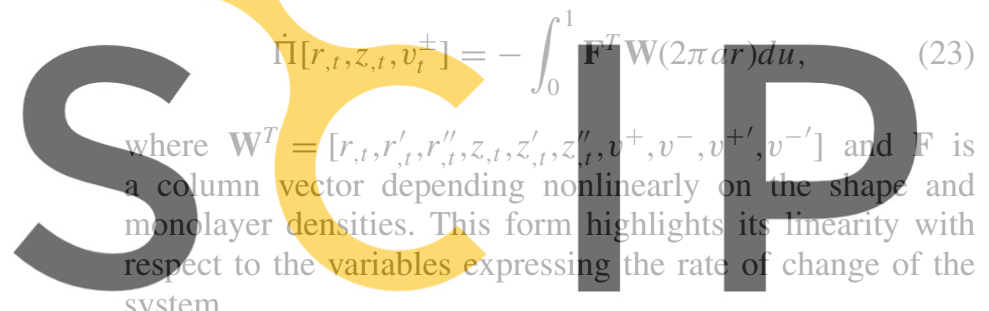
system.

A common assumption when studying bilayer vesicles is

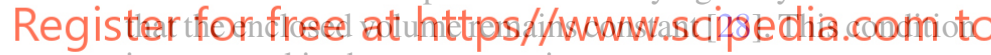
is expressed in the present setting as

$$
0=C^{\mathrm{vol}}\left[r_{, t}, z, t\right]=\dot{V}=\int_{0}^{1}\left(-z^{\prime} r_{, t}+r^{\prime} z_{, t}\right)(2 \pi r) d u .
$$

Minimizing the Lagrangian subject to the constraints, one can find the velocities $\left\{r_{, t}, z_{,}, v^{ \pm}\right\}$at each configuration $\left\{r, z, \rho^{ \pm}\right\}$. Then, the surface parametrization can be integrated in time from $\left\{r_{t, t}, z_{t}\right\}$, and the monolayer densities from the continuity equation.

\section{NUMERICAL APPROXIMATION}

In this section, we discretize the governing equations with a Galerkin method. We represent numerically shape and the physical fields using $B$ splines and derive the space-discretized form of the governing equations, a system of differentialalgebraic equations.

\section{A. Spacial semi-discretization}

The generating curve of the axisymmetric surface is represented numerically as a $B$-spline curve

$$
\{r(u, t), z(u, t)\} \approx \sum_{I=1}^{N} B_{I}(u) \underbrace{\left\{r_{I}(t), z_{I}(t)\right\}}_{\mathbf{P}_{I}(t)},
$$

where $B_{I}(u)$ are the $B$-spline basis functions [29] defined on the interval $[0,1]$, and $\left\{r_{I}(t), z_{I}(t)\right\}$ is the position of the $I$ th control point of the $B$-spline curve at instant $t$. Similarly, the density field is represented as

$$
\rho^{ \pm}(u ; t) \approx \sum_{I=1}^{\tilde{N}} \tilde{B}_{I}(u) \rho_{I}^{ \pm}(t) .
$$

We then have

$$
\begin{aligned}
\left\{r_{, t}, z_{, t}\right\} & \approx \sum_{I=1}^{N} B_{I}(u) \underbrace{\left\{\dot{r}_{I}(t), \dot{z}_{I}(t)\right\}}_{\dot{\mathbf{P}}_{I}(t)}, \\
\rho_{, t}^{ \pm} & \approx \sum_{I=1}^{\tilde{N}} \tilde{B}_{I}(u) \dot{\rho}_{I}^{ \pm}(t) .
\end{aligned}
$$

Finally, the tangential velocity of each monolayer can be numerically expressed by

$$
v^{ \pm}(u ; t) \approx \sum_{I=1}^{\hat{N}} \hat{B}_{I}(u) v_{I}^{ \pm}(t) .
$$

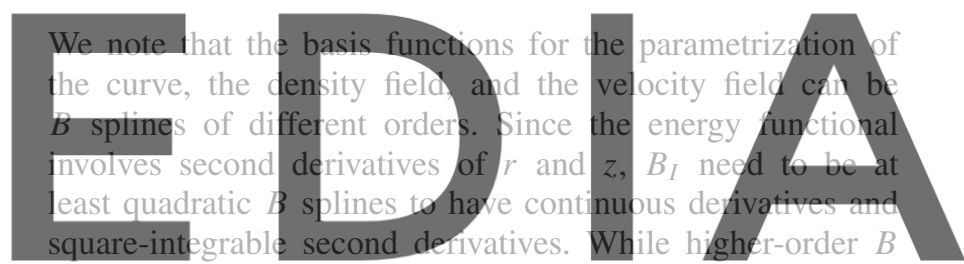

splines have superior accuracy, their computational cost is also higher, because the bandwidth of the dissipation matrices

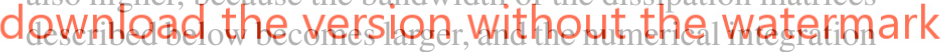

requires more quadrature points. We found the results to be quite insensitive to the degree of the basis functions, and in the calculations we consider cubic $B$ splines for the shape and for the tangential velocity fields, and quadratic $B$ splines for the density. This combination provides stable and accurate results at a reasonable computational cost.

\section{B. Discretized form of the governing equations}

Plugging these representations into Eqs. (18)-(20) and (23), and making the Lagrangian stationary with respect to $\dot{\mathbf{P}}_{I}$, $v_{I}^{ \pm}$, and the Lagrange multipliers $\boldsymbol{\Lambda}$, we obtain a system of differential algebraic equations (DAEs),

$$
\begin{aligned}
\mathbf{D}_{\text {visc }}\left[\begin{array}{c}
\dot{\mathbf{P}} \\
\mathbf{V}
\end{array}\right]+\left[\begin{array}{cc}
\widehat{\mathbf{D}} & \mathbf{0} \\
\mathbf{0} & \mathbf{D}_{\mathrm{fr}}
\end{array}\right]\left[\begin{array}{c}
\dot{\mathbf{P}} \\
\mathbf{V}
\end{array}\right]+\mathbf{Q} \mathbf{\Lambda} & =\boldsymbol{f}(\boldsymbol{\rho}, \mathbf{P}), \\
\mathbf{Q}^{T}\left[\begin{array}{c}
\dot{\mathbf{P}} \\
\mathbf{V}
\end{array}\right] & =\mathbf{0},
\end{aligned}
$$

where the global column arrays $\mathbf{P}, \mathbf{V}$, and $\rho$ collect all the degrees of freedom. All the dissipation and constraint matrices depend nonlinearly on $\mathbf{P}$. The constraint matrix $\mathbf{Q}$ encodes boundary conditions and possibly the fixed volume constraint. The membrane dissipation matrix $\mathbf{D}_{\text {visc }}$ follows from Eq. (19) 
and takes the form

$$
\mathbf{D}_{\mathrm{visc}}=\int_{\Gamma} \mathbf{B}^{T} \mathbf{A B} d S,
$$

where $\mathbf{B}$ is a matrix whose entries are $B_{I}, \hat{B}_{I}$, and $\hat{B}_{I}^{\prime}$, the integrals are performed by Gaussian numerical quadrature, and the global matrix is filled with the standard assembly process in finite elements [22]. Similarly, the inter-monolayer friction matrix is assembled from local matrices of the form

$$
\left(\mathbf{D}_{\mathrm{fr}}\right)_{I J}=b_{m} \int_{\Gamma}\left[\begin{array}{cc}
\hat{B}_{I} & 0 \\
0 & \hat{B}_{I}
\end{array}\right]\left[\begin{array}{cc}
1 & -1 \\
-1 & 1
\end{array}\right]\left[\begin{array}{ll}
\hat{B}_{J} & 0 \\
0 & \hat{B}_{J}
\end{array}\right] d S,
$$

and for the parametrization dissipation we have

$$
(\widehat{\mathbf{D}})_{I J}=\frac{\hat{\mu}}{S} \int_{\Gamma} \frac{1}{a^{2}}\left[\begin{array}{cc}
B_{I} & 0 \\
0 & B_{I}
\end{array}\right]\left[\begin{array}{cc}
r^{\prime 2} & r^{\prime} z^{\prime} \\
r^{\prime} z^{\prime} & z^{\prime 2}
\end{array}\right]\left[\begin{array}{cc}
B_{J} & 0 \\
0 & B_{J}
\end{array}\right] d S .
$$

From Eq. (23) we have

$$
\boldsymbol{f}=\int_{\Gamma} \overline{\mathbf{B}}^{T} \mathbf{F} d S
$$

where now $\overline{\mathbf{B}}$ is filled with $B_{I}, B_{I}^{\prime}, B_{I}^{\prime \prime}, \hat{B}_{I}$, and $\hat{B}_{I}^{\prime}$

The continuity equation is a partial differential equation governing the evolution of the density field. Due to the

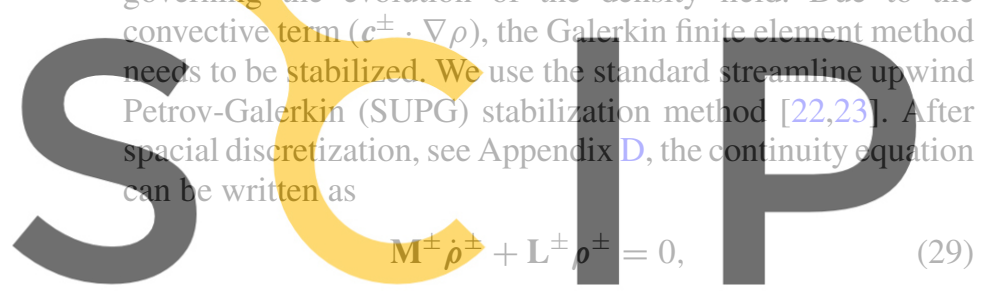

where the $\mathbf{M}$ and $\mathbf{L}$ matrices depend on $\dot{\mathbf{P}}$ and $\mathbf{V}$

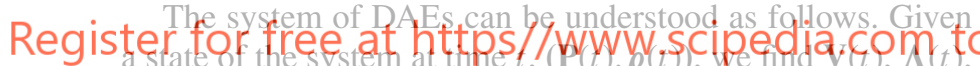

and $\dot{\mathbb{P}}(t)$ from Eq. (28). We then use Eq. (29) to compute $\dot{\rho}(t)$. Thus, we can formally express the rate of change of the system as $(\dot{\mathbf{P}}(t), \dot{\rho}(t))=\boldsymbol{G}(\mathbf{P}(t), \rho(t))$. We integrate forward in time this ODE with specialized semi-implicit ODE solvers for stiff problems [30].

\section{NUMERICAL RESULTS}

We exercise now the model in selected applications of interest. We first revisit the problem of tether extension as a validation of our model and simulations. We then present a suite of examples that illustrate the diversity of dynamical regimes of lipid bilayers, all of which bear biological relevance. We have carefully checked the convergence of the numerical approximation by mesh refinement. We find that the numerical method is robust to the order of the $B$-spline basis functions, to the parametrization dissipation coefficient $\hat{\mu}$, and to the stabilization parameter $\tau$ of the SUPG method. In the examples presented here, we use at most 100 basis functions for each of the unknowns, $r, z, \rho^{ \pm}$, and $v^{ \pm}$.

\section{A. Material parameters and time scales}

Since our model includes several energetic and kinetic coupled mechanisms, the choice of material parameters $(\kappa$, $K_{s}, \mu, b_{m}$, and $d$ ) plays an important role in the behavior of the system, and there is no canonical nondimensionalization of the equations ( $\lambda$ does not play a major role). Note carefully that $K_{s}$ and $\mu$ are monolayer parameters, and therefore a factor of 2 is needed to relate the model to some reported measurements. We set the distance between the bilayer midsurface and the monolayer neutral surface to $d=1 \mathrm{~nm}$. It is useful to define nondimensional parameters comparing elastic and dissipative mechanisms respectively. On the one hand, $\epsilon=\kappa /\left(2 K_{s} d^{2}\right)$ takes values on the order of 1 for lipid bilayers. On the other hand, $\alpha=2 \mu /\left(b_{m} d^{2}\right)$ shows a larger variability. With reported values for the membrane shear viscosity $10^{-10}<2 \mu<5 \times$ $10^{-9} \mathrm{Js} / \mathrm{m}^{2}$ and for the inter-monolayer friction $10^{8}<b_{m}<$ $10^{9} \mathrm{Js} / \mathrm{m}^{4}, \alpha$ ranges between 0.1 and 50 . Here we consider $\alpha=1$. In all the simulations, we consider $\kappa=10^{-19} \mathrm{~J}, 2 K_{s}=$ $0.1 \mathrm{~J} / \mathrm{m}^{2}, b_{m}=10^{9} \mathrm{Js} / \mathrm{m}^{4}$, and $2 \mu=10^{-9} \mathrm{Js} / \mathrm{m}^{2}[9,31]$.

We discuss next a number of time scales that govern the dynamics under different circumstances. Let us denote by $\bar{\rho}$ the density average and by $\hat{\rho}$ the density difference between the two monolayers, normalized by $\rho_{0}$. At fixed shape, the gradients in the density difference $\hat{\rho}$ have been shown to evolve according to a diffusion equation with diffusivity $D=K_{s} / b_{m}$ [14], which introduces a relaxation time scale $t_{1}=\bar{S} / D$, where $\bar{S}$ is the relevant area, here of a density difference disturbance. Density differences can also relax by changing shape and creating curvature, see Fig. 5, which when

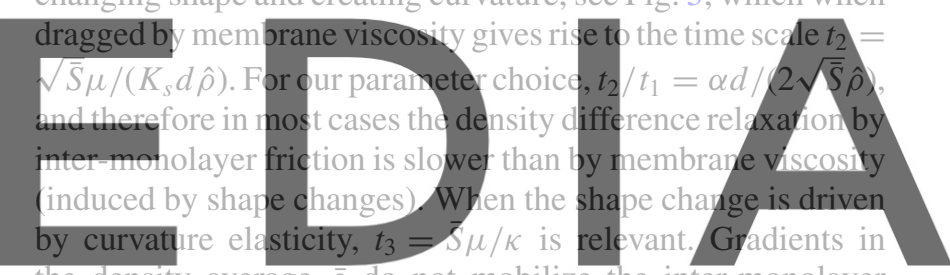

the density average $\bar{\rho}$ do not mobilize the inter-monolayer friction and exhibit an extremely fast characteristic time scale

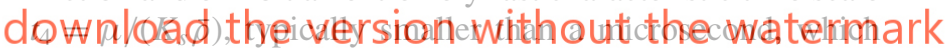
needs to be resolved by the simulations at initial stages. When the dynamics mobilize bending energy and inter-monolayer friction, the relevant time scale is $t_{5}=\bar{S} d^{2} b_{m} / \kappa$. Some of these time scales have been considered previously, e.g., in Ref. [15].

\section{B. Dynamics of tether formation and extrusion}

The statics of bilayer tubular structures has been extensively studied from a theoretical point of view. One can easily estimate the equilibrium configuration of a tether pulled out of a giant vesicle under constant surface tension $\sigma$ by minimizing the energy of a uniform cylindrical membrane $E=\pi \kappa L / r+2 \pi \sigma r L$ with respect to the tube length $L$ and radius $r$, leading to expressions for the equilibrium radius $r=\sqrt{\kappa / 2 \sigma}$, and for the static force needed hold the tube

$$
f^{s}=\pi \kappa / r+2 \pi \sigma r=2 \pi \sqrt{2 \kappa \sigma} .
$$

The equilibrium force during quasistatic tether nucleation, eventually converging to $f^{s}$, has been studied theoretically $[32,33]$.

The dynamical features of tether growth and retraction have been investigated theoretically and experimentally in many studies [14,34,35]. Evans and Yeung [14] considered a giant vesicle kept at constant tension $\sigma$ by a micropipette, and pulled out a tether with an adhesive microbead at constant velocity 
$\dot{L}$. They provided a theoretical estimation of the pulling force $f$ when the tube area is much smaller than that of the vesicle,

$$
\begin{aligned}
f= & 2 \pi\left(r \sigma+\frac{\kappa}{2 r}\right)+2 \pi\left(\frac{2 K_{s} d^{2}}{R^{2}}\right) L \\
& +2 \pi \underbrace{\left[2 \mu-3 \eta_{w} r+\left(4 b_{m} d^{2}\right) \ln \frac{R}{r}\right]}_{\eta_{\text {eff }}} \dot{L},
\end{aligned}
$$

where $\eta_{w}$ denotes the water viscosity, $R$ denotes the radius of the vesicle, and $\eta_{\text {eff }}$ denotes the effective viscosity of the tether. The first line is an elastic force $f_{\text {elast }}$, with a first term corresponding to the constant static force $f^{s}$ and a second term, proportional to the tether's length, accounting for the global area difference between the monolayers. The second line is the rate-dependent force $f_{\text {rate }}$, which groups all hydrodynamic effects, i.e., the bulk and membrane viscous forces and the slippage between the monolayers at the tether's neck. For large vesicles, the nonlocal elastic term can be neglected, and the viscous forces are overwhelmingly dominated by inter-monolayer friction.

We test our simulations against these theoretical predictions. We remove the nonlocal effect by pulling a tether out of a large enough planar membrane disk of radius $R$ with constant surface tension $\sigma$ boundary condition, and uncoupled

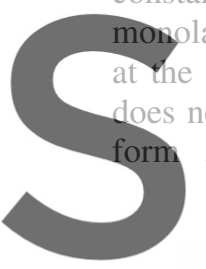
layers (other than by frictiona boundary of the disk $(u$ ot move $r_{t}(1)=0$ and $\pi R \sigma v^{ \pm}(1)$ is added
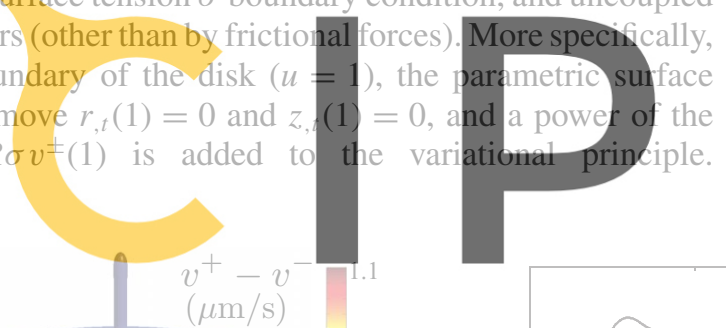

The tangential velocities at the boundary of the domain $v^{ \pm}(1)$ are free, and are taken into account in the balance of mass since the system exchanges mass with its surroundings. Figure 2 shows a tether nucleation and extraction at constant rate. The color map, Fig. 2(a), shows the velocity difference between the leaflets, visualizing the inter-monolayer slippage at the tether's neck. The radius of the tube during this process ranges between 71.1 and $76.3 \mathrm{~nm}$. By stopping the extraction, we check that the small deviation from the static radius $(r=70.7 \mathrm{~nm})$ is due to dynamical effects. The maximum pulling force, about $1.17 f^{s}$, is attained for a critical length $0.32 R$, a value known to depend on the radius of the vesicle or the disk [33]. The steady state pulling force from our simulations is $f^{\infty}=1.12 f^{s}$, where the $12 \%$ deviation from $f^{s}$ is explained by the additional terms in Eq. (31). To assess their magnitude, we annihilate in the computations the friction coefficient first, and then the membrane viscosity. The resulting drops in the tether force are shown in the inset of Fig. 2(b). Most of the rate-dependent part of the force is due, as expected, to inter-monolayer friction. The membrane shear viscosity contribution is only $f_{u}=0.00712 f^{s}$, very close to the the theoretical estimation from Eq. (31), $0.00707 f^{s}$, which shows the quantitative agreement between the simulations and the available theoretical predictions. We can further understand the phenomenon by tracking elastic energies and dissipation

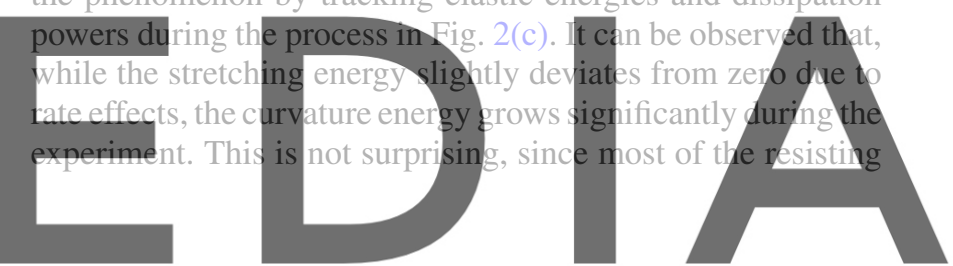

Register for free at https//www.scipedia.com to download the version without the watermark
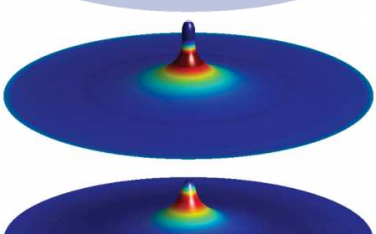

(b)

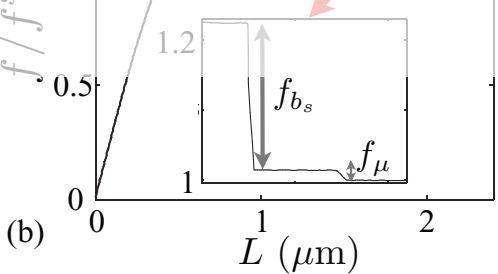

(c)
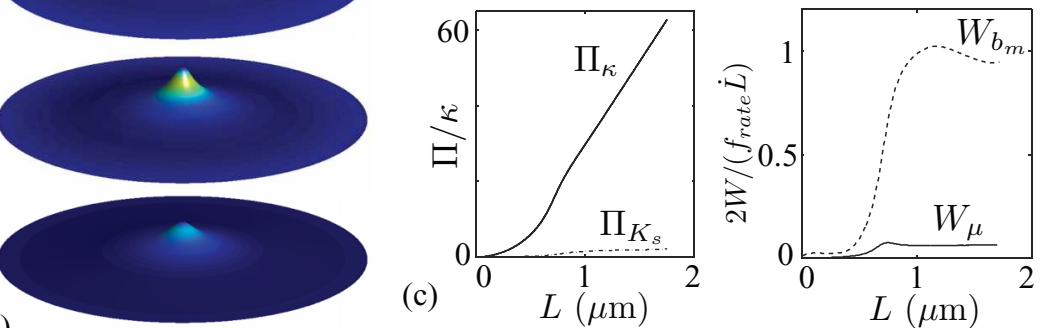

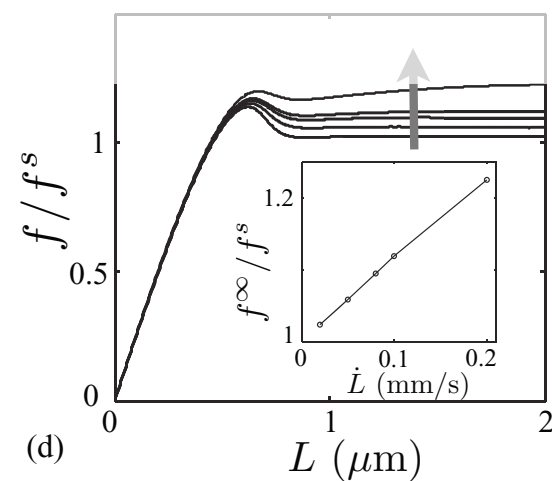

(d) (a)

FIG. 2. (Color online) Nucleation and extension of a tether out of a planar disk of radius $2 \mu \mathrm{m}$ kept at constant surface tension $(\sigma=$ $\sigma^{+}+\sigma^{-}=10^{-4} K_{s}$ ). All figures but the right plot are for a pulling rate $\dot{L}=0.1 \mathrm{~mm} / \mathrm{s}$. (a) Selected snapshots with a color map of the inter-monolayer slippage velocity. Once the tether is fully formed, the process reaches a steady state, where the shape, slippage at the neck, and the radius of the tether do not change. (b) Force-extension curve normalized by the static tether force in Eq. (30). The end of the process and the inset highlight the effect of membrane shear viscosity and inter-monolayer friction on the dynamical part of the force. (c) Evolution of the elastic energy $(\Pi)$ and dissipation power $(2 W)$ components during the process. (d) Effect of the loading rate for $\dot{L}=0.02,0.05,0.08,0.1,0.2 \mathrm{~mm} / \mathrm{s}$. The arrow indicates increasing rate. The inset shows the normalized steady-state force $f^{\infty}$ as a function of strain rate. From the slope of this curve, we can compute the effective tether viscosity $\eta_{\text {eff }}$ in Eq. (31). 
force is static and explained by $f_{s}$. Before the tube has fully nucleated, the growth of the curvature energy is quadratic, and after the buckling event that forms the tether, it grows linearly, with a slope that we confirm is very close (within 1\%) to half $f^{s}$ as predicted by Eq. (30). It can also be observed that most of the dissipation in this process is due to inter-monolayer friction. It can be easily seen from Eq. (31) that the viscoelastic time constant for this process is $t_{5}=\bar{S} d^{2} b_{m} / \kappa=r L d^{2} b_{m} / \kappa$. Recalling the expression for the tube radius, the characteristic tether pulling velocity at which viscous forces are comparable to elastic forces is $\dot{L}_{c}=L / t_{5}=\sqrt{2 \kappa \sigma} /\left(4 b_{m} d^{2}\right) \approx 0.25 \mathrm{~mm} / \mathrm{s}$, in agreement with our results, Fig. 2(d).

The left plot in Fig. 2 shows the effect of pulling rate on the resisting force. The rate of the process changes slightly the buckling point, but more importantly the steady state force $f^{\infty}$ increases with rate. From Eq. (31), the slope of $f^{\infty}$ as a function of $\dot{L}$ should provide the effective tether viscosity $\eta_{\text {eff }}$ (see the figure inset), which has been examined experimentally $[34,36]$. We find that, for the largest rate, the relation slightly departs from linearity, due to the dynamically induced elastic force resulting from $\Pi_{K_{\mathrm{s}}}$ mentioned above.

In summary, this example validates our model and simulations in a well understood situation, and provides a detailed picture of the rate effects during such process. Tether extension may behave quite differently, for instance for vesicles pulled

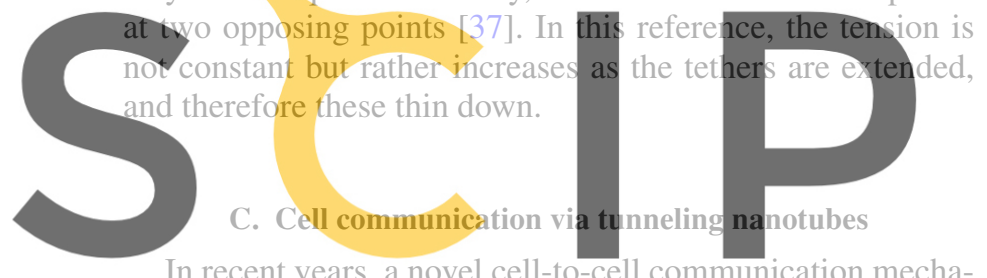

In recent years, a novel cell-to-cell communication mechanism mediated by membrane nanotubes bridging animal cells

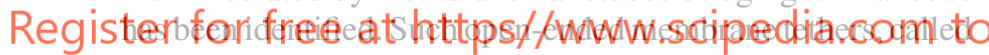
tunneling nanotubes (TNTs), have been shown to facilitate physiological functions in cell-to-cell communication during health and disease [38-40]. For instance, there is evidence that HIV-1 can spread quickly via TNTs between cells in the human immune system [41].

TNTs have diameters $2 r$ ranging from 50 to $200 \mathrm{~nm}$ and lengths $L$ of up to tens of micrometers. Transport of objects through TNTs has been shown in vitro to be mostly driven by the difference of surface tension $\Delta \sigma$ between the connected liposomes, and not by the internal pressure difference $\Delta p$ [42]. Indeed, the fluid velocity profile inside the tube can be expressed as $v(s)=v_{l}-v_{0}\left[1-(s / r)^{2}\right]$, where $s$ is the radial position, $v_{l}$ is the inner monolayer lipid velocity driven by the tension difference $\Delta \sigma$, and $v_{0}=r \Delta p /\left(2 \eta_{w} L\right)$ is the amplitude of the backward flow due to the pressure difference. For long thin tubes, $v_{0}$ can be neglected. As before, the radius of the connecting tube can be estimated by $r=\sqrt{\kappa / 2 \bar{\sigma}}$ where $\bar{\sigma}$ is the average tension between the two cells. This phenomenon, driven by gradients in the surface tension, can thus be interpreted as a Marangoni effect.

We consider a long tether $(L=8 \mu \mathrm{m})$ bridging two giant vesicles modeled by two circular disks of radius $R=3 \mu \mathrm{m}$, see Fig. 3 (left). Initially, we obtain the static equilibrium by fixing the same surface tension at the top and bottom boundaries. Then, we induce the lipid flow by increasing the tension difference $\Delta \sigma$, while their average $\bar{\sigma}$ is fixed. If the process is performed very slowly, we can assume that the membrane flow is at steady state. We visualize the

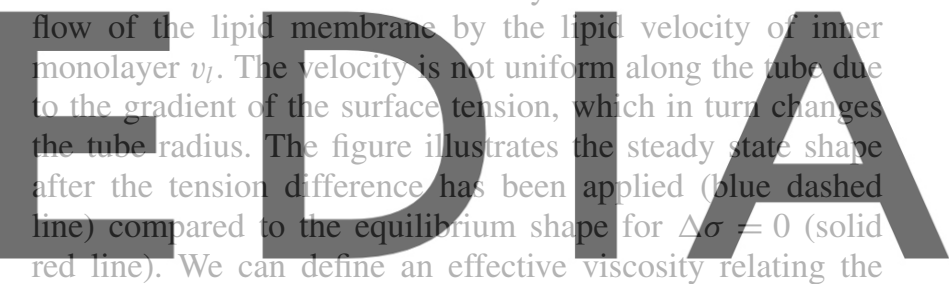

velocity of the lipids to the tension difference, $\Delta \sigma=\eta_{\mathrm{eff}} v_{l}$.

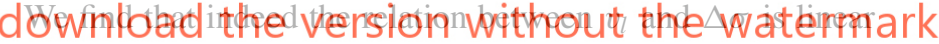
in a wide range of tension differences of up to $20 \%$ of $\bar{\sigma}$. Figure 3 (middle) shows how the effective viscosity increases with average tension (as the tube radius decreases). As the
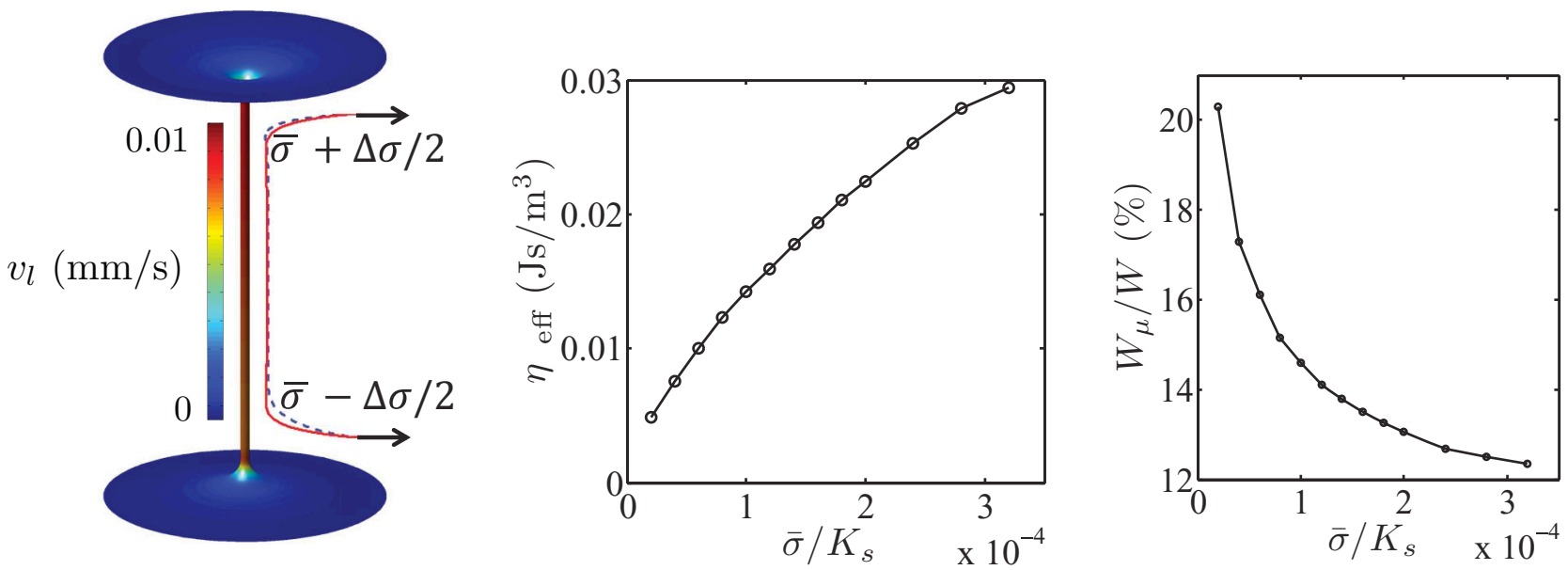

FIG. 3. (Color online) Cell-to-cell communication via tunneling nanotubes. Geometry and lipid velocity field of the inner monolayer at steady state for $\Delta \sigma=1.36 \times 10^{-4} \mathrm{mN} / \mathrm{m}$ and $\bar{\sigma}=0.005 \mathrm{mN} / \mathrm{m}=10^{-4} K_{s}$, corresponding to $r=100 \mathrm{~nm}$ (left). The shape change as a result of the tension difference is highlighted by the dashed blue generating curve, to be compared with the static shape (solid red line). Effective viscosity as a function of the average tension between the two connected membrane patches $\bar{\sigma}$ (middle). Fraction of the membrane shear dissipation relative to the total dissipation as a function of $\bar{\sigma}$ (right). 

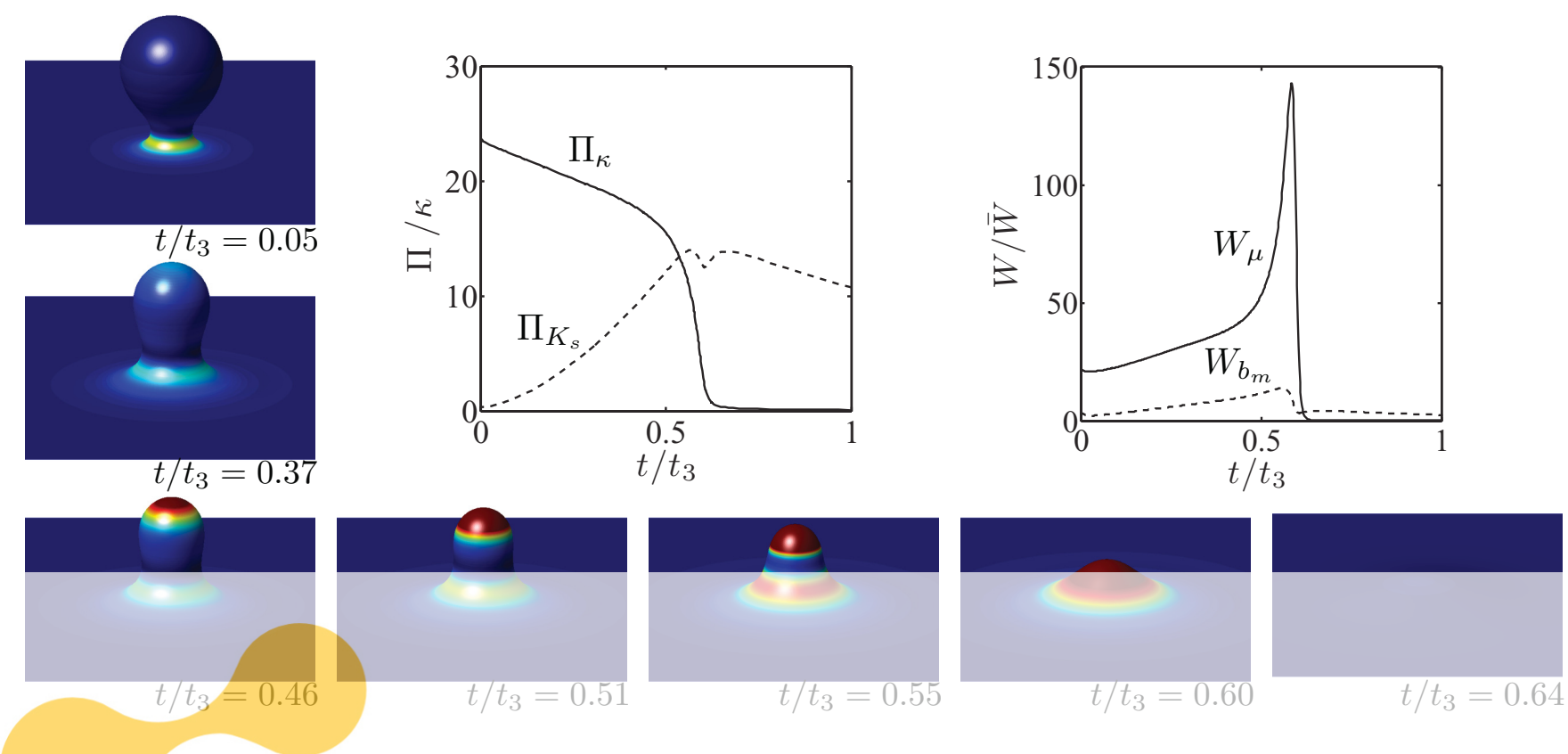

FIG. 4. (Color online) Relaxation dynamics of a bud of radius $R=0.34 \mu \mathrm{m}$ connected to a tense planar membrane disk $(\sigma=0.006 \mathrm{mN} / \mathrm{m})$. The color map on the snapshots shows the membrane dissipation power density in the same scale during the process. The left plot shows the curvature and stretching elastic energies during the process, while the right plot shows the membrane shear and inter-monolayer friction dissipation powers, in nondimensional te
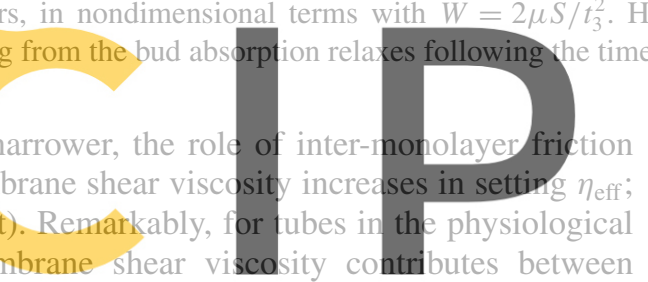

$10 \%$ and $20 \%$ of the total dissipation, and is therefore not Registegligible for quantitative predictions. Focusing on the main

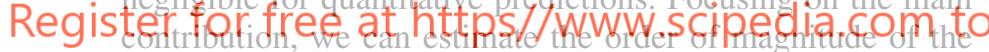
effective viscosity by scaling arguments as $\eta_{\text {eff }} \approx b_{m} d^{2} / r=$ $b_{m} d^{2} \sqrt{2 \bar{\sigma} / \kappa}$, leading to a characteristic time for transport across the TNT of $t_{6}=b_{m} d^{2} L \sqrt{2 \bar{\sigma} / \kappa} / \Delta \sigma$.

\section{Bud relaxation}

We consider now the relaxation of a vesicle that has formed a fusion pore with a planar membrane disk under constant tension. Figure 4 shows selected snapshots of the process, by which the bud is eventually absorbed completely and the bilayer disk becomes planar. Here, the curvature forces are the main elastic mechanism driving the process (see Fig. 4, left plot). The applied membrane tension also drives the absorption. The dominant dissipative mechanism is the membrane shear dissipation (see Fig. 4, right plot), and therefore, as expected, the relaxation dynamics are governed by $t_{3} \approx 3.6 \mathrm{~ms}$. It can be observed that, during the bud absorption, some amount of stretching elasticity is stored since initially the bud and the planar bilayer were at their equilibrium lipid density. The density difference created as the bud disappears then dissipates slowly through inter-monolayer friction in a time scale of $t_{1} \approx 15 \mathrm{~ms}$.

The process proceeds first rather slowly, with the membrane shear dissipation concentrated at the neck of the bud. Recalling Eqs. (10) and (11), we can interpret that this localized shear

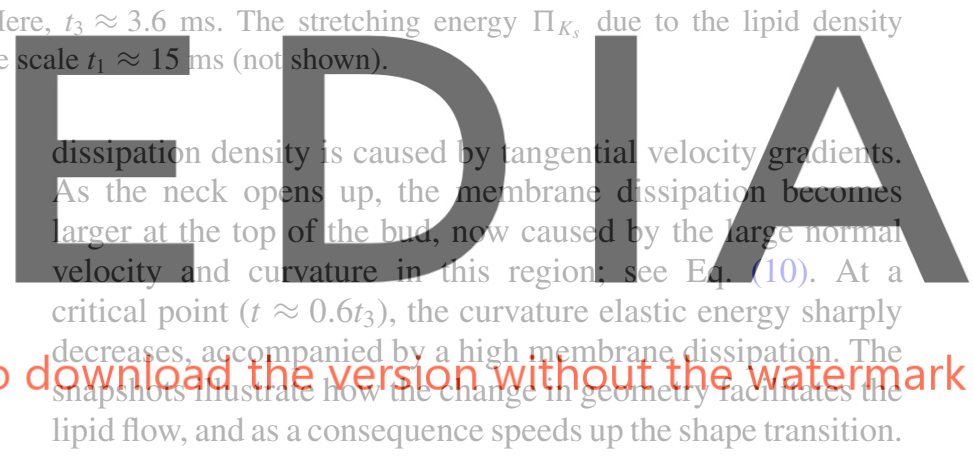

E. Density asymmetry excitations in vesicles

Organelles and cells are often placed out of equilibrium by localized density disturbances caused by a myriad of physicochemical phenomena. Proteins or polymers inserting into the bilayer, effectively changing the lipid packing, can induce shape changes $[1,43]$. Lipid molecules can locally change their shape and size, for instance under localized $\mathrm{pH}$ alterations, leading to transient shape changes [11,12]. Lipid translocation between the monolayers driven by flippases can induce budding $[15,44]$. Figure 5 illustrates how the insertion/extraction of a molecule in one monolayer locally increases/decreases the lipid density, mobilizing inter-monolayer slippage and/or creating curvature.

In this study, we focus on deflated, spheroidal, axisymmetric vesicles initially in equilibrium. Since lipid membranes are almost area preserving, a completely spherical vesicle is too tight to allow for shape changes. The excess area relative to the vesicle volume is conventionally quantified by the reduced volume, defined as the ratio between the enclosed volume and the volume of a sphere with equal surface area. We start with a prolate spheroid, with a reduced volume of 0.98 , although analogous results are found with oblate shapes as long as there is available excess area to change shape 

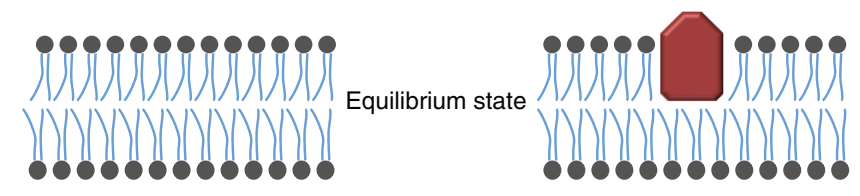

Protein insertion
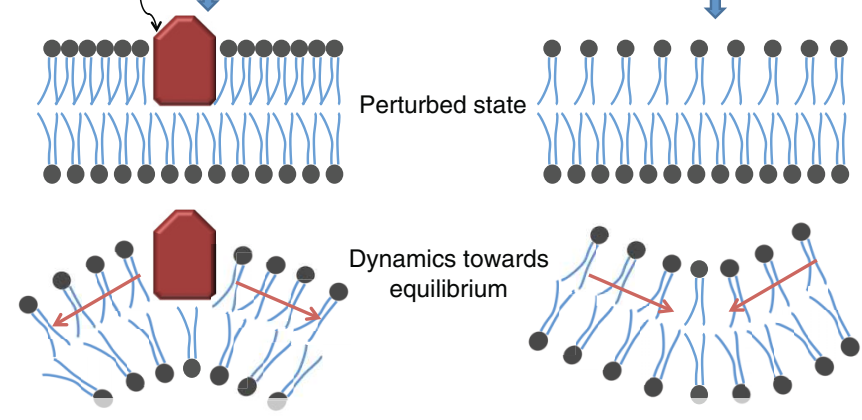

FIG. 5. (Color online) Hlustration of how a molecule insertion/extraction in a monolayer disturbs the lipid density asymmetrically. The lipid density contrast across the monolayers can be relaxed either by flowing lipids away from/towards the disturbance (thin red arrows), or by curving the bilayer to form buds/invaginations

without stretching significantly the bilayer. In fact, the last example presented here exhibits a transition between a prolate and an oblate configuration, triggered by a localized density asymmetry. We consider a small vesicle of radius $R=100 \mathrm{~nm}$, a large vesicle $(R=2 \mu \mathrm{m})$ and a giant vesicle $(R=4 \mu \mathrm{m})$ We chose a different number of lipids in each mondlayes so that the equilibrium density in the neutral surface of the monolayers is close to $\rho_{0}$. To accomplish this, we set initially the projected densities to $\rho^{ \pm}=\rho_{0}(1 \pm 2 d / R)$, and then find the equilibrium state by minimizing $\Pi$ subject to the mass

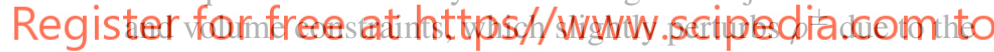
nonuniform curvature of the prolate shape.

Once the initial state has been prepared, we locally perturb the lipid density of the outer monolayer, and examine numerically the relaxation dynamics. Specifically, the lipid density at the neutral surface of the outer monolayer $\hat{\rho}^{+}$is disturbed with the profile $\delta \rho \times f(\phi)$ shown in Fig. 6, where $\phi \in[0, \pi]$ is the spherical angle of the vesicle domain. We choose the width of the disturbance and its amplitude as $w=10 \%$ and $\delta \rho=0.05 \rho_{0}$.

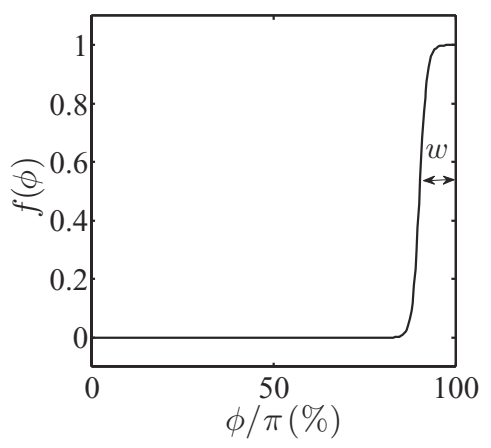

FIG. 6. Profile of the density perturbation on the outer monolayer. The density at the neutral surface $\hat{\rho}^{+}$is disturbed by $\delta \rho \times f(\phi)$. With our choice of width of the profile, $w=10 \%$, the disturbance occupies about $2.5 \%$ of the area of the vesicle.
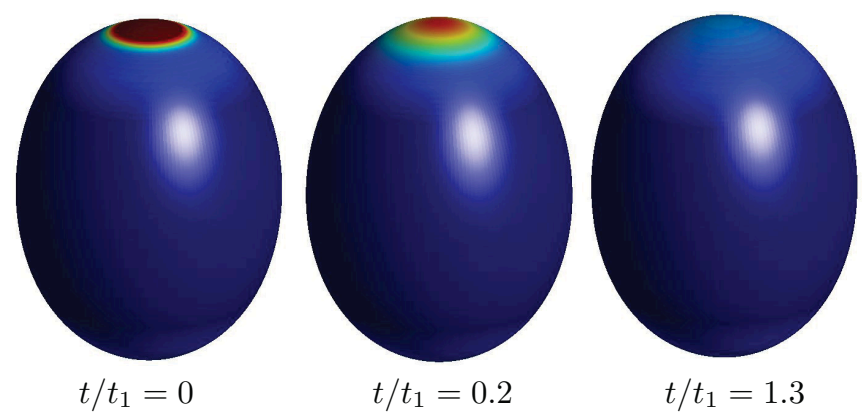

FIG. 7. (Color online) Relaxation dynamics of the small vesicle ( $R=100 \mathrm{~nm}, t_{1}=0.06 \mathrm{~ms}, t_{2}=0.02 \mathrm{~ms}$ ). The density disturbance diffuses without noticeable shape changes. The color maps represent the difference between the monolayer lipid densities at the neutral surface, $\hat{\rho}^{+}-\hat{\rho}^{-}$, where red corresponds to $\delta \rho$ and blue to 0 .

Such density perturbation creates a gradient of both density average and density difference of magnitude $\delta \rho / 2$ [8]. The density average disturbance relaxes, dragged by membrane shear viscosity, extremely fast in a time scale commensurate to $t_{4}$. This initial event needs to be captured by the simulations, although we do not report on it. Then, the density difference disturbance relaxes by local curvature. Such a localized shape change is dragged mainly by membrane shear viscosity in

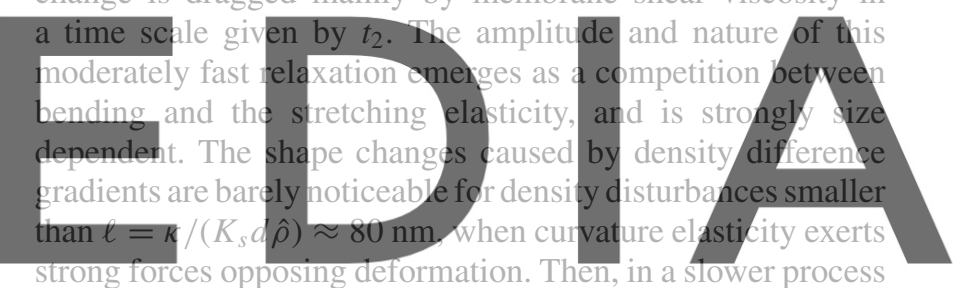
dictated by $t_{1}$, the density difference is dissipated through inter-

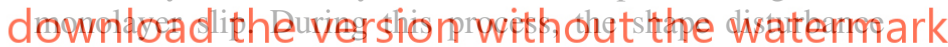
adapts very quickly to the diffusing density difference, and eventually disappears.

Figures 7-9 show the shape and density evolution of perturbed prolate vesicles of different radii as they relax towards a new equilibrium state. For the small vesicle, Fig. 7, the density deviations hardly produce any shape deformation due to the large resistance of bending elasticity relative to stretching elasticity as the size of the disturbance is smaller than $\ell$. The aggregated density diffuses in the lateral direction dragged by inter-monolayer slippage. The time scale for density diffusion is $t_{1}=\bar{S} b_{m} / K_{s} \approx 0.06 \mathrm{~ms}$, where $\bar{S}=2 \pi(1-\cos w \phi) R^{2}$ is the area of the asymmetry patch. The small shape perturbation occurs at a faster time scale given by $t_{2} \approx 0.36 t_{1}$. For the large vesicle, Fig. 8 , the resisting elastic forces due to curvature are much less relevant, and in a very fast time scale commensurate to $t_{2} \approx 2 \times 10^{-2} t_{1}$, a geometric feature of significant amplitude relaxes part of the stretching elastic energy. Due to the time scale separation, the shape adapts almost instantaneously to equilibrate stretching and curvature forces as the density slowly relaxes by intermonolayer friction. The transient bud eventually disappears as the density fully equilibrates. A similar behavior can be observed for the giant vesicle, Fig. 9, where now the dynamics are complicated by a richer shape landscape at the current ratio between the disturbance size and $\ell$. As before, in the very initial stages given by $t_{2}$, a large amplitude bud forms 

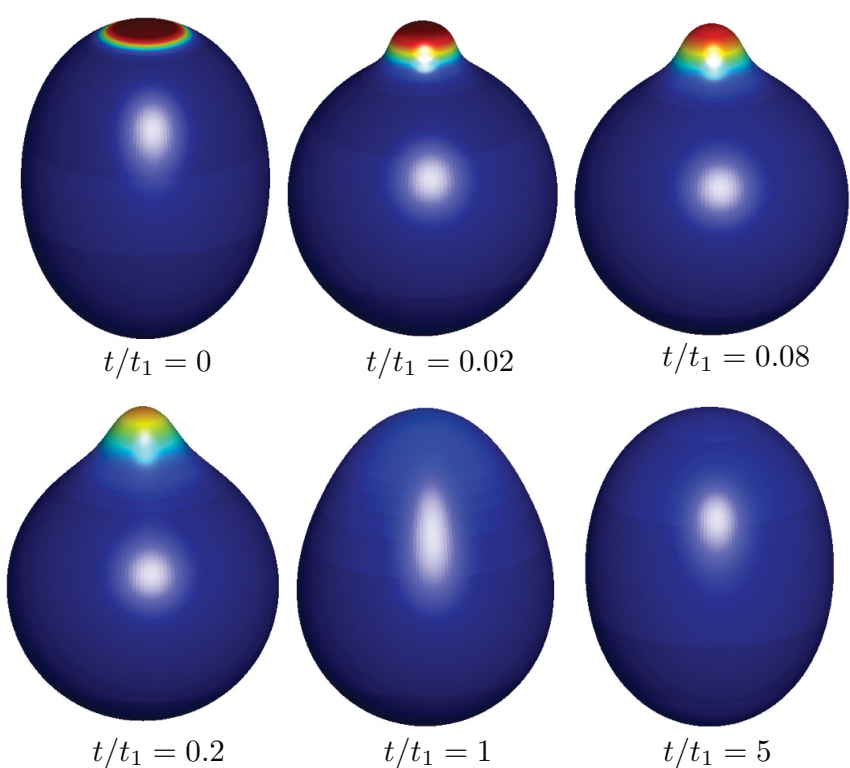

FIG. 8. (Color online) Relaxation dynamics of the large vesicle ( $R=2 \mu \mathrm{m}, t_{1}=25 \mathrm{~ms}, t_{2}=0.44 \mathrm{~ms}$ ). At early stages, the density disturbance relaxes by forming a bud, which then disappears and the density difference diffuses by inter-monolayer friction. The color maps represent the difference between the monolayer lipid densities at the neutral surface, $\hat{\rho}^{+}-\hat{\rho}^{-}$, where red corresponds to $\delta \rho$ and blue to 0 .

at the density disturbance. As the density diffuses, there is a shape transition by which the bud elongates into a short tube at $t \approx 0.12 t_{1}$. This fast shape transition relaxes abruptly the stretching energy and slightly increases the curvature energy, resulting in a net decrease of the total energy. This abrupt shape change is accompanied by a spike in the membrane shear viscosity (see Fig. 10). This figure also shows the initial spike in membrane dissipation associated with $t_{2}$.

Subsequently, the elongated protrusion pearls, and the number of pearls decreases in steps until there is a single vesicle connected to the mother vesicle by a narrow neck. The color map in Fig. 9 shows how the density difference is quantized by the size of the pearls. Interestingly, these complex shapes transiently trap the density asymmetries, and slow down significantly the density relaxation by inter-monolayer slip. The full relaxation takes $t \approx 10 t_{1}$. This example shows the intimate coupling between lipid flow and shape dynamics. Previous insightful theoretical work [15] captures the essential physics, but is restricted to a shape ansatz that does not agree with the shapes we find and that are reported in some experiments.

Similar phenomena have been observed experimentally, for instance by locally anchoring polymers or amphiphilic molecules to oblate lipid vesicles [43]. Once polymers anchor to the bilayer, they induce curvature both by increasing the area of the monolayer, and by a local deformation of the bilayer (spontaneous curvature). The anchoring molecules then diffuse on the membrane. Figure 11 shows the experimental observations from [43], which are qualitatively in agreement with our simulations. Similarly, a localized $\mathrm{pH}$ gradient can change the repulsion between the lipid head-groups of the exposed monolayer and form the metastable pearled tubes
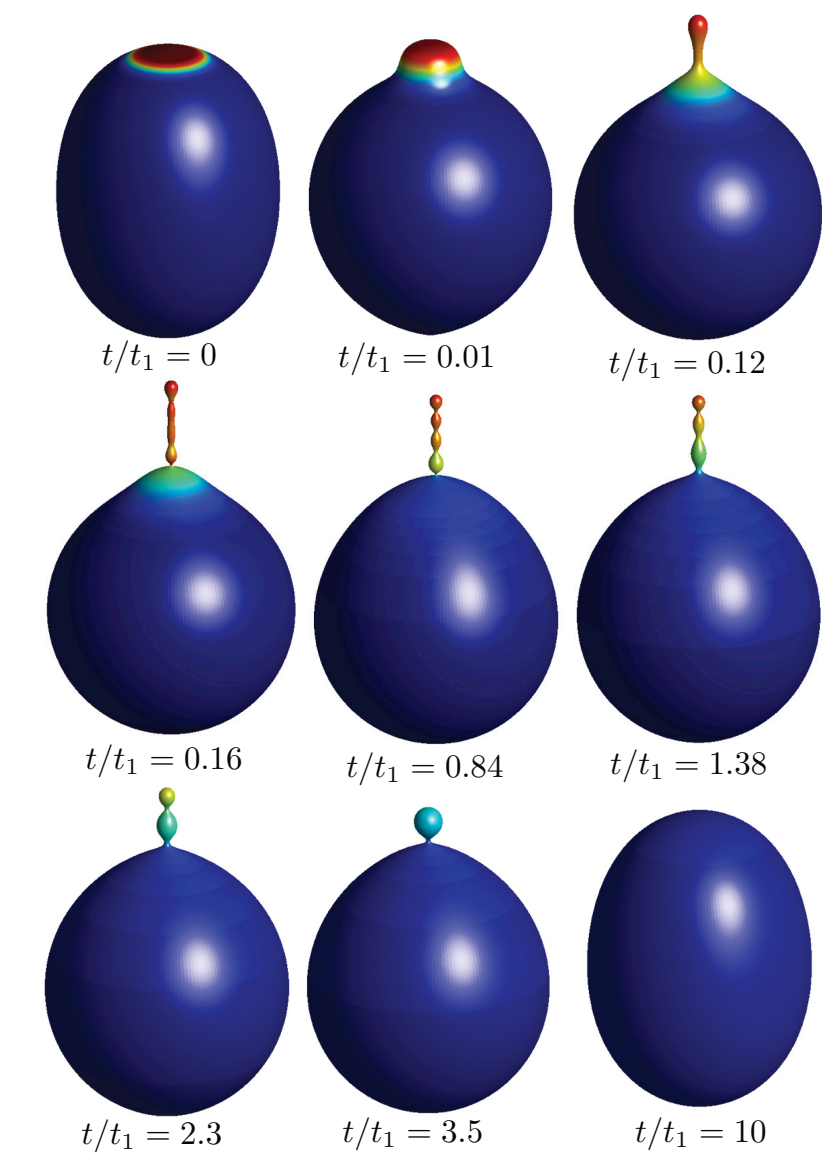

FIG. 9. (Color online) Relaxation dynamics of the giant vesicle ( $R=4 \mu \mathrm{m}, t_{1}=98 \mathrm{~ms}, t_{2}=0.88 \mathrm{~ms}$ ). The density difference relaxation by inter-monolayer slippage is slowed down significantly by the dramatic shape changes, which transiently trap the density asymmetries. At a later time $\left(t / t_{1} \approx 10\right)$, the spherical bud is absorbed by the mother vesicle [45]. The color maps represent the difference between the monolayer lipid densities at the neutral surface, $\hat{\rho}^{+}-\hat{\rho}^{-}$, where red corresponds to $\delta \rho$ and blue to 0 .

$[11,12]$. Such asymmetries have been modeled mathematically by considering a transient spontaneous curvature [46], an area difference [11], or a density asymmetry parameter [12].

Stomatocyte morphologies are often observed in vesicles at equilibrium, and have been explained on the basis of area difference or spontaneous curvature [47]. However, the dynamical studies of the stomatocyte formation are limited. The reversible dynamics of membrane invaginations has been studied by a local acid injection [11]. Going back to Fig. 5, we explore the transient formation of stomatocytes by removing lipids locally from the outer leaflet. We consider a large vesicle $R=2 \mu \mathrm{m}$, with a density disturbance characterized by $\delta \rho=$ $-0.05 \rho_{0}$ and $w=10 \%$. Figure 12 shows the stomatocyte formation by relaxation of density difference through shape changes at a time scale $t_{2}$, and then the full relaxation through inter-monolayer slippage at a time scale slightly longer than $t_{1}$. Again, the second stage of the relaxation dynamics is slowed down by the complex shapes adopted by the vesicle. Interestingly, these dynamics provide a pathway between two 

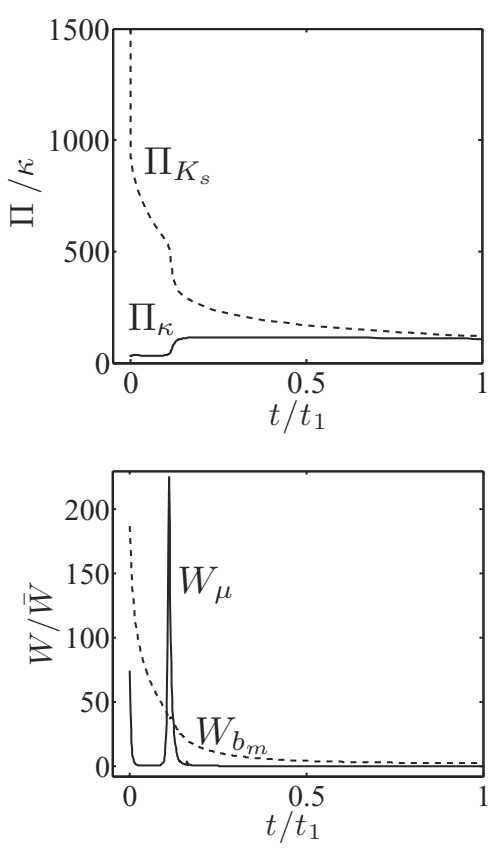

FIG. 10. Elastic energy (top) and dissipation power (bottom) during the relaxation of the giant vesicle, $R=4 \mu \mathrm{m}$. The dissipation power is normalized by $\bar{W}=\bar{S} b_{m} d^{2} /\left(\hat{\rho} t_{1}^{2}\right)$. After a fast initial relaxation of the density difference by budding, which leaves as a signature a first spike in the membrane shear dissipation, the dynamics are dictated by the inter-monolayer friction. Yet, at $t \approx 0.12 t_{1}$, the density difference field is such that a rapid shape transition from a bud to an elongated protrusion occurs, leaving another large spike of membrane shear dissipation.

metastable branches of the equilibrium phase diagram, prolates and oblates.

We finally note that our results quantitatively depend on the reduced volume, the magnitude of the density disturbance, and its size relative to the vesicle size. A systematic characterization of the dynamical behavior of such density disturbances is the topic of current work. In the present study, we have not considered larger vesicles, since then the effect of the bulk fluid viscosity, ignored here for simplicity, may become important.
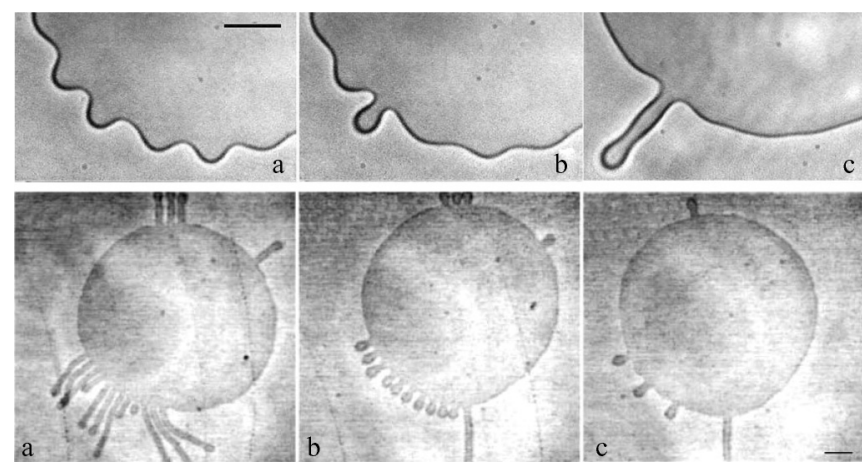

FIG. 11. Experimental observations from Ref. [43]; tube and bud formation after a local injection of polymers in the vicinity of a flaccid giant vesicle. The first row shows the nucleation of buds and their transient elongation into tubes. The second row shows further elongation and the subsequent retraction of the bud-tube shapes.
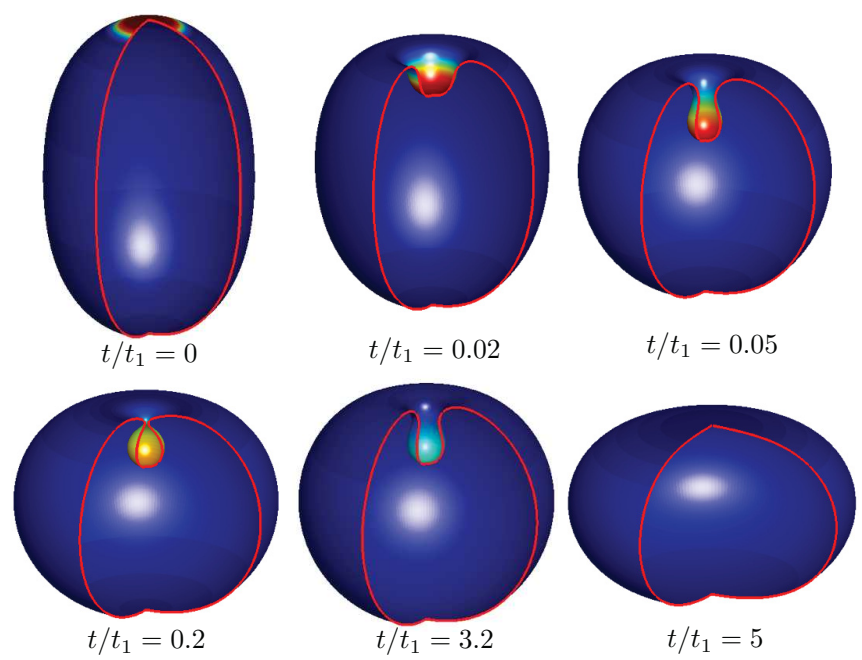

FIG. 12. (Color online) Stomatocyte formation from a large vesicle of radius $R=2 \mu \mathrm{m}$, locally perturbed by an inverse density asymmetry. During the relaxation of the density disturbance, the vesicle switches from prolate to oblate.

\section{CONCLUSIONS}

We have proposed a comprehensive dynamical continuum model for lipid bilayers to investigate out-of-equilibrium phenomena, and implemented it numerically. Our simulations, restricted to axisymmetry, are to our knowledge the first to consider general and finite shape and lipid density changes. This model allows us to study very complex dynamical events of biological relevance, without resorting to simplifying assumptions on the magnitude of the disturbances, the kinds of shapes the bilayer can adopt, or the relative importance of the different phenomena. After validating the model with the well understood membrane tethering, we have presented a gallery of examples, which highlights the versatility and generality of the model in describing very different processes involving lipid hydrodynamics and shape dynamics. These examples show that some usual assumptions can oversimplify the response of bilayers to various stimuli, and illustrate a wide diversity of dynamical regimes. In tether pulling, the viscoelastic behavior is given by the bending elasticity and the inter-monolayer friction. In the intercell communication through tunneling tubes, membrane tension gradients, together with inter-monolayer friction and membrane shear viscosity, set the time scale of transport. In the relaxation dynamics of a bud absorption into a planar bilayer, two time scales are operative: the faster one, given by bending elasticity and the membrane shear viscosity, attenuates the geometry of the bud, while the slower one, given by stretching elasticity and inter-monolayer friction, relaxes a residual density difference between the monolayers. This latter time scale is the dominant one in the relaxation of localized asymmetric density disturbances in vesicles, which also possess a faster time scale given by stretching elasticity and membrane shear viscosity. It is important to note that these observations are specific to our parameter choice (material parameters such as $\alpha$, size, applied tension,...), and in general the dynamics depend very much on the details. Furthermore, our simulations illustrate the highly nontrivial effect of the bilayer shape on the dynamics. 
Thus, the proposed model and simulation method is a valuable tool to interpret experiments and interrogate hypothesis about biological phenomena mediated by bilayers. We are currently using it to investigate the dynamics of confined bilayers [21], as well as the dynamics of bilayers in the presence of membrane proteins.

\section{ACKNOWLEDGMENTS}

We acknowledge helpful discussions with Antonio DeSimone, and the support of the European Research Council under the European Community's 7th Framework Programme (FP7/2007-2013)/ERC Grant Agreement No. 240487. M.R. acknowledges the support of the Spanish Government through Grant No. BES-2008-003274. M.A. acknowledges the support received through the prize "ICREA Academia" for excellence in research, funded by the Generalitat de Catalunya.

\section{APPENDIX A: SURFACE STRESS TENSOR}

To determine the Cauchy surface stress tensor, we follow the method presented in Ref. [48] based on the principle of virtual work; see also Ref. [49]. Suppose that the free energy of the bilayer is given by

$$
\Pi=\int_{\Gamma} f(\boldsymbol{g}, \boldsymbol{k}) d S,
$$

where $f(\boldsymbol{g}, \boldsymbol{k})$ is the free energy per unit area. Then, the general expression for the elastic surface stress tensor is

$$
\sigma^{a b}=\underbrace{\mathscr{T}^{a b}+\mathscr{H}^{a c} k_{c}^{b}}_{\sigma_{\|}^{a b}}-\underbrace{\nabla_{c} \mathscr{H}^{a c} n^{b}}_{\sigma_{\perp}^{a b}},
$$

where

$$
\begin{aligned}
\mathscr{T}^{a b} & =\frac{2}{\sqrt{g}} \frac{\partial(\sqrt{g} f)}{\partial g_{a b}}, \\
\mathscr{H}^{a b} & =\frac{\partial f}{\partial k_{a b}},
\end{aligned}
$$

and $g$ is the determinant of the metric tensor. We have changed the sign in the definition of this stress tensor as compared to the references above to be consistent with the Doyle-Ericksen formula [50] and the usual convention, by which positive tractions are tensile. Note also our different sign convention in defining the second fundamental form. When dotted with a unit tangential vector to the surface and normal to a curve on it $\boldsymbol{l}$, this tensor provides the tractions along this curve $l^{c} g_{c a} \sigma_{\|}^{a b}$ and $l^{c} g_{c a} \sigma_{\perp}^{a b}$, tangential and normal to the surface [49].

We have

$$
\begin{gathered}
\frac{\partial \sqrt{g}}{\partial g_{a b}}=\frac{1}{2} \sqrt{g} g^{a b}, \quad 2 \frac{\partial H}{\partial g_{a b}}=-k^{a b}, \\
2 \frac{\partial H}{\partial k_{a b}}=g^{a b}, \quad \frac{\partial \rho^{ \pm}}{\partial g_{a b}}=-\frac{1}{2} \rho^{ \pm} g^{a b} .
\end{gathered}
$$

where the last equation comes from differentiating the following statement of balance of mass $\rho^{ \pm} \sqrt{g}=$ cst. In the present case,

$$
f=\kappa\left(2 H-C_{0}\right)^{2} / 2+K_{s}\left(\rho^{ \pm} / \rho_{0} \mp 2 d H-1\right)^{2} / 2,
$$

leading to

$$
\begin{aligned}
\sigma_{\|}^{a b}= & f g^{a b}-\kappa\left(2 H-C_{0}\right) k^{a b} \\
& -K_{s}\left(\frac{\rho^{ \pm}}{\rho_{0}}-1 \mp 2 d H\right)\left(\frac{\rho^{ \pm}}{\rho_{0}} g^{a b} \pm d k^{a b}\right)
\end{aligned}
$$

which is symmetric as expected, and to

$$
\sigma_{\perp}^{a b}=-2 \kappa \nabla^{a} H n^{b} \pm d K_{s} \nabla^{a}\left(\frac{\rho^{ \pm}}{\rho_{0}} \mp 2 d H\right) n^{b} .
$$

In both expressions, there is an implied summation on the two monolayers contributions. The stretching term usually dominates the in-plane stress tensor. For moderate density variations and bilayer curvature, this results in the familiar expression

$$
\boldsymbol{\sigma}_{\|} \approx-K_{s}\left(\frac{\hat{\rho}^{ \pm}}{\rho_{0}}-1\right) \boldsymbol{g} .
$$

Also note that for uniform bilayers, e.g., spheres or tubes with uniform lipid density, $\sigma_{\perp}=0$.

\section{APPENDIX B: MEMBRANE DISSIPATION FOR AXISYMMETRIC SURFACES}

We first compute the following terms of Eq. (11) for axisymmetric surfaces, dropping the superindex \pm for simplicity:

$$
\begin{aligned}
& \boldsymbol{d}: \boldsymbol{d}=\left(\frac{1}{a} v^{\prime}\right)^{2}+\left(\frac{r^{\prime}}{a r} v\right)^{2}-\frac{2}{a} v_{n}\left(\frac{b}{a^{3}} v^{\prime}+\frac{z^{\prime} r^{\prime}}{a r^{2}} v\right) \\
& +\left(4 H^{2}-2 K\right) v_{n}^{2} \\
& =\frac{1}{a^{2}} v^{\prime 2}+\left(\frac{r^{\prime}}{a r}\right)^{2} v^{2}+\frac{2 z^{\prime} b}{a^{5}} r_{, t} v^{\prime}+\frac{2 z^{\prime 2} r^{\prime}}{r^{2} a^{3}} r_{, t} v \\
& -\frac{2 r^{\prime} b}{a^{5}} z_{, t} v^{\prime}-\frac{2 r^{\prime 2} z^{\prime}}{r^{2} a^{3}} z_{, t} v+\frac{\left(4 H^{2}-2 K\right) z^{\prime 2}}{a^{2}} r_{, t}{ }^{2} \\
& +\frac{\left(4 H^{2}-2 K\right) r^{\prime 2}}{a^{2}} z_{, t}^{2}-\frac{2\left(4 H^{2}-2 K\right) r^{\prime} z^{\prime}}{a^{2}} r_{, t} z_{, t}, \\
& (\operatorname{trd})^{2}=\left(\frac{(r v)^{\prime}}{r a}-2 v_{n} H\right)^{2} \\
& =\frac{1}{a^{2}} v^{\prime 2}+\left(\frac{r^{\prime}}{a r}\right)^{2} v^{2}+\frac{4 z^{\prime} H}{a^{2}} r_{, t} v^{\prime}+\frac{4 z^{\prime} r^{\prime} H}{r a^{2}} r_{, t} v \\
& -\frac{4 r^{\prime} H}{a^{2}} z, t v^{\prime}-\frac{4 r^{\prime 2} H}{r a^{2}} z_{, t} v+2 \frac{r^{\prime}}{r a^{2}} v v^{\prime} \\
& +\frac{4 H^{2} z^{\prime 2}}{a^{2}} r_{, t}^{2}+\frac{4 H^{2} r^{\prime 2}}{a^{2}} z_{, t}^{2}-\frac{8 H^{2} r^{\prime} z^{\prime}}{a^{2}} r_{, t} z_{, t} .
\end{aligned}
$$

By substituting the above relations into Eq. (11), we can identify the nonzero components of the matrix $\mathbf{A}$ as

$$
\begin{aligned}
& A_{11}=4 \mu \frac{\left(4 H^{2}-2 K\right) z^{\prime 2}}{a^{2}}+8 \lambda \frac{H^{2} z^{\prime 2}}{a^{2}}, \\
& A_{12}=-4 \mu \frac{\left(4 H^{2}-2 K\right) r^{\prime} z^{\prime}}{a^{2}}-8 \lambda \frac{H^{2} r^{\prime} z^{\prime}}{a^{2}},
\end{aligned}
$$




$$
\begin{aligned}
& A_{13}=A_{14}=2 \mu \frac{z^{\prime 2} r^{\prime}}{r^{2} a^{3}}+2 \lambda \frac{z^{\prime} r^{\prime} H}{r a^{2}}, \\
& A_{15}=A_{16}=2 \mu \frac{z^{\prime} b}{a^{5}}+2 \lambda \frac{z^{\prime} H}{a^{2}}, \\
& A_{22}=4 \mu \frac{\left(4 H^{2}-2 K\right) r^{\prime 2}}{a^{2}}+8 \lambda \frac{H^{2} r^{\prime 2}}{a^{2}}, \\
& A_{23}=A_{24}=-2 \mu \frac{r^{\prime 2} z^{\prime}}{r^{2} a^{3}}-2 \lambda \frac{r^{\prime 2} H^{\prime}}{r a^{2}} \\
& A_{33}=A_{44}=(2 \mu+\lambda)\left(\frac{r^{\prime}}{a r}\right)^{2}, \\
& A_{25}=A_{26}=-2 \mu \frac{r^{\prime} b}{a^{5}}-2 \lambda \frac{r^{\prime} H}{a^{2}}, \\
& A_{35}=A_{46}=\lambda \frac{r^{\prime}}{r a^{2}}, \\
& A_{55}=A_{66}=(2 \mu+\lambda) \frac{1}{a^{2}} .
\end{aligned}
$$

\section{APPENDIX C: VARIATION OF ELASTIC ENERGY FOR AXISYMMETRIC SURFACES}

Recalling definitions and the continuity equation, simple calculations show that

$$
\begin{aligned}
H_{, t}= & \frac{\partial H}{\partial r} r_{, t}+\frac{\partial H}{\partial r^{\prime}} r_{, t}^{\prime}+\frac{\partial H}{\partial r^{\prime \prime}} r_{, t}^{\prime \prime}+\frac{\partial H}{\partial z^{\prime}} z_{, t}^{\prime}+\frac{\partial H}{\partial z^{\prime \prime}} z_{, t}^{\prime \prime}, \\
a_{, t}= & \frac{r^{\prime}}{a} r_{, t}^{\prime}+\frac{z^{\prime}}{a} z_{, t}^{\prime}, \\
\rho_{, t}^{ \pm}= & -\left(\frac{\rho^{ \pm} r^{\prime}}{r a}+\frac{\rho^{ \pm^{\prime}}}{a}\right) v^{ \pm}-\frac{\rho^{ \pm}}{a} v^{ \pm \prime} \\
& +\left(\frac{\rho^{ \pm \prime} r^{\prime}}{a^{2}}-\frac{2 \rho^{ \pm} z^{\prime} H}{a}\right) r_{, t}+\left(\frac{\rho^{ \pm \prime} z^{\prime}}{a^{2}}+\frac{2 \rho^{ \pm} r^{\prime} H}{a}\right) z_{, t}
\end{aligned}
$$

and

$$
\begin{aligned}
& 2 \frac{\partial H}{\partial r}=-\frac{z^{\prime}}{a r^{2}}, \quad 2 \frac{\partial H}{\partial r^{\prime}}=\frac{z^{\prime \prime}}{a^{3}}-\frac{3 b r^{\prime}}{a^{5}}, \quad 2 \frac{\partial H}{\partial r^{\prime \prime}}=-\frac{z^{\prime}}{a^{3}}, \\
& 2 \frac{\partial H}{\partial z^{\prime}}=-\frac{r^{\prime \prime}}{a^{3}}-\frac{3 b z^{\prime}}{a^{5}}+\frac{r^{\prime 2}}{a^{3} r}, \quad 2 \frac{\partial H}{\partial z^{\prime \prime}}=\frac{r^{\prime}}{a^{3}} .
\end{aligned}
$$

\section{APPENDIX D: SPACE DISCRETIZATION OF THE CONTINUITY EQUATION}

Following a standard SUPG stabilization method, the weight functions for the continuity equation are $\left(\tilde{B}_{I}+\tau c\right.$. $\left.\nabla \tilde{B}_{I}\right)$, where $\tau=h /(2\|\boldsymbol{c}\|)$ and $h$ is the grid size. We rewrite the continuity equation in Eq. (17) as

$$
\rho_{, t}^{ \pm}+s^{ \pm} \rho^{ \pm}+c^{ \pm} \rho^{ \pm^{\prime}}=0
$$

where

$$
\begin{aligned}
& s^{ \pm}=\frac{v^{ \pm} r^{\prime}}{r a}+\frac{v^{ \pm \prime}}{a}+\frac{2 z^{\prime} H r_{, t}}{a}-\frac{2 r^{\prime} H z_{, t}}{a}, \\
& c^{ \pm}=\frac{v^{ \pm}}{a}-\frac{r^{\prime} r_{, t}}{a^{2}}-\frac{z^{\prime} z_{, t}}{a^{2}} .
\end{aligned}
$$

By replacing Eqs. (25) and (26) in the equation above and multiplying by the SUPG weight functions, we obtain for each monolayer the discrete form of the continuity equation

$$
\left(\mathbf{M}^{ \pm}+\mathbf{M}^{s t b \pm}\right) \dot{\rho}^{ \pm}+\left(\mathbf{L}_{1}^{ \pm}+\mathbf{L}_{2}^{ \pm}+\mathbf{L}_{1}^{s t b \pm}+\mathbf{L}_{2}^{s t b \pm}\right) \boldsymbol{\rho}^{ \pm}=0,
$$

where

$$
\begin{aligned}
M_{I J}^{ \pm} & =\int_{\Gamma} \tilde{B}_{I} \tilde{B}_{J} d S, \quad M_{I J}^{s t b \pm}=\int_{\Gamma} \tau \frac{c^{ \pm}}{a} \tilde{B}_{I}^{\prime} \tilde{B}_{J} d S, \\
L_{1 J}^{ \pm} & =\int_{\Gamma} \tilde{B}_{I} s^{ \pm} \tilde{B}_{J} d S, \quad L_{2}^{ \pm}=\int_{\Gamma} \tilde{B}_{I} c^{ \pm} \tilde{B}_{J}^{\prime} d S, \\
L_{1 J}^{s t b \pm} & =\int_{\Gamma} \tau \frac{c^{ \pm}}{a} \tilde{B}_{I}^{\prime} s^{ \pm} \tilde{B}_{J} d S, \\
L_{2}^{s t b \pm}{ }_{I J} & =\int_{\Gamma} \tau \frac{c^{ \pm}}{a} \tilde{B}_{I}^{\prime} c^{ \pm} \tilde{B}_{J}^{\prime} d S .
\end{aligned}
$$

Note that the $\mathbf{M}$ and $\mathbf{L}$ matrices depend on $\dot{\mathbf{P}}$ and $\mathbf{V}$.
[1] Y. Shibata, J. Hu, M. M. Kozlov, and T. A. Rapoport, Annu. Rev. Cell Dev. Biol. 25, 329 (2009).

[2] P. G. Saffman and M. Delbrück, Proc. Natl. Acad. Sci. USA 72 3111 (1975).

[3] A. Rustom, R. Saffrich, I. Markovic, P. Walther, and H. Gerdes, Science 303, 1007 (2004).

[4] H. Sprong, P. van der Sluijs, and G. van Meer, Nat. Rev. Mol. Cell Biol. 2, 504 (2001).

[5] M. Staykova, D. Holmes, C. Read, and H. Stone, Proc. Natl. Acad. Sci. USA 108, 9084 (2011).

[6] F. Julicher and R. Lipowsky, Phys. Rev. Lett. 70, 2964 (1993).

[7] L. Miao, U. Seifert, M. Wortis, and H.-G. Döbereiner, Phys. Rev. E 49, 5389 (1994).

[8] U. Seifert, Adv. Phys. 46, 13 (1997).

[9] R. Dimova, S. Aranda, N. Bezlyepkina, V. Nikolov, K. A. Riske, and R. Lipowsky, J. Phys. Condens. Matter 18, S1151 (2006).
[10] P. Devaux, Biochimie 82, 497 (2000)

[11] N. Khalifat, N. Puff, S. Bonneau, J.-B. Fournier, and M. I. Angelova, Biophys. J. 95, 4924 (2008).

[12] J. B. Fournier, N. Khalifat, N. Puff, and M. I. Angelova, Phys. Rev. Lett. 102, 018102 (2009).

[13] U. Seifert and S. A. Langer, S. A., Europhys. Lett. 23, 71 (1993).

[14] E. Evans and A. Yeung, Chem. Phys. Lipids 73, 39 (1994).

[15] P. Sens, Phys. Rev. Lett. 93, 108103 (2004).

[16] M. Arroyo and A. DeSimone, Phys. Rev. E 79, 031915 (2009).

[17] M. Rahimi, A. DeSimone, and M. Arroyo (unpublished).

[18] I. R. Cooke, K. Kremer, and M. Deserno, Phys. Rev. E 72, 011506 (2005).

[19] B. Reynwar, G. Illya, V. Harmandaris, M. Müller, K. Kremer, and M. Deserno, Nature (London) 447, 461 (2007).

[20] M. Klein and W. Shinoda, Science 321, 798 (2008). 
[21] M. Staykova, M. Arroyo, M. Rahimi, and H. Stone (unpublished).

[22] T. Belytschko, W. Liu, and B. Moran, Nonlinear Finite Elements for Continua and structures, Vol. 36 (Wiley, New York, 2000).

[23] J. Donea and A. Huerta, Finite Element Methods for Flow Problems (John Wiley \& Sons, Chichester, UK, 2003).

[24] D. Marsh, Biophys. J. 93, 3884 (2007).

[25] L. Scriven, Chem. Eng. Sci. 12, 98 (1960).

[26] H. Goldstein, C. Poole, and J. Safko, Classical Mechanics (Addison-Wesley, Reading, MA, 2001).

[27] M. Arroyo, A. Desimone, and L. Heltai (unpublished).

[28] U. Seifert and R. Lipowsky, in Structure and Dynamics of Membranes, edited by R. Lipowsky and E. Sackmann, Vol. 1 of Handbook of Biological Physics (Elsevier Science, New York, 1995), Chap. 8.

[29] L. Piegl and W. Tiller, The NURBS book (Springer Verlag, Berlin, 1997).

[30] L. F. Shampine, M. W. Reichelt, and J. A. Kierzenka, Siam Rev. 41, 538 (1999).

[31] R. Dimova, U. Seifert, B. Pouligny, S. Forster, and H. Dobereiner, Eur. Phys. J. E 7, 241 (2002).

[32] T. R. Powers, G. Huber, and R. E. Goldstein, Phys. Rev. E 65 , 041901 (2002).

[33] I. Derényi, F. Julicher, and J. Prost, Phys. Rev. Lett. 88, 238101 (2002).

[34] Z. Li, B. Anvari, M. Takashima, P. Brecht, J. H. Torres, and W. E. Brownell, Biophys. J. 82, 1386 (2002).

[35] O. Rossier, D. Cuvelier, N. Borghi, P. Puech, I. Derenyi, A. Buguin, P. Nassoy, and F. Brochard-Wyart, Langmuir 19, 575 (2003).

[36] R. Waugh, Biophys. J. 38, 29 (1982).
[37] H. J. Lee, E. L. Peterson, R. Phillips, W. S. Klug, and P. A. Wiggins, Proc. Natl. Acad. Sci. USA 105, 9253 (2008).

[38] A. Rustom, R. Saffrich, I. Markovic, P. Walther, and H. Gerdes, Science 303, 1007 (2004).

[39] H. Gerdes and R. Carvalho, Curr. Opin. Cell Biol. 20, 470 (2008).

[40] G. Dubey and S. Ben-Yehuda, Cell 144, 590 (2011).

[41] S. Sowinski, C. Jolly, O. Berninghausen, M. Purbhoo, A. Chauveau, K. Kohler, S. Oddos, P. Eissmann, F. Brodsky, C. Hopkins et al., Nat. Cell Biol. 10, 211 (2008).

[42] R. Karlsson, M. Karlsson, A. Karlsson, A. Cans, J. Bergenholtz, B. Akerman, A. Ewing, M. Voinova, and O. Orwar, Langmuir 18, 4186 (2002).

[43] I. Tsafrir, Y. Caspi, M. A. Guedeau-Boudeville, T. Arzi, and J. Stavans, Phys. Rev. Lett. 91, 138102 (2003).

[44] A. Papadopulos, S. Vehring, I. Lopez-Montero, L. Kutschenko, M. Stoeckl, P. Devaux, M. Kozlov, T. Pomorski, and A. Herrmann, J. Biol. Chem. 282, 15559 (2007).

[45] See supplemental material at http://link.aps.org/supplemental/ 10.1103/PhysRevE.86.011932 for a movie of the dynamics.

[46] F. Campelo and A. Hernandez-Machado, Phys. Rev. Lett. 100, 158103 (2008).

[47] S. Svetina, Chem. Phys. Chem. 10, 2769 (2009).

[48] R. Capovilla and J. Guven, J. Phys. A: Math. Gen. 35, 6233 (2002).

[49] M. M. Müller, Ph.D. thesis, Johannes Gutenberg-Universitat in Mainz, 2007 (unpublished).

[50] J. E. Marsden and T. Hughes, The Mathematical Foundations of Elasticity (Prentice-Hall, Englewood Cliffs, NJ, 1983). 